Preprint typeset in JHEP style. - HYPER VERSION

hep-th/0310182

AEI-2003-080

\title{
Matching Higher Conserved Charges for Strings and Spins
}

\author{
Gleb Arutyunov and Matthias Staudacher \\ Max-Planck-Institut für Gravitationsphysik, Albert-Einstein-Institut \\ Am Mühlenberg 1, D-14476 Golm, Germany \\ Email: agleb,matthias@aei.mpg.de
}

\begin{abstract}
We demonstrate that the recently found agreement between one-loop scaling dimensions of large dimension operators in $\mathcal{N}=4$ gauge theory and energies of spinning strings on $A d S_{5} \times S^{5}$ extends to the eigenvalues of an infinite number of hidden higher commuting charges. This dynamical agreement is of a mathematically highly intricate and non-trivial nature. In particular, on the gauge side the generating function for the commuting charges is obtained by integrable quantum spin chain techniques from the thermodynamic density distribution function of Bethe roots. On the string side the generating function, containing information to arbitrary loop order, is constructed by solving exactly the Bäcklund equations of the integrable classical string sigma model. Our finding should be an important step towards matching the integrable structures on the string and gauge side of the AdS/CFT correspondence.
\end{abstract}

KEYwORDS: AdS-CFT Correspondence; Duality in Gauge Field Theories. 


\section{Contents}

1. Introduction and summary 1

2. Higher one-loop charges in gauge theory 5

2.1 Generalities 5

2.2 Folded case 8

2.3 Circular case 10

3. Higher semiclassical charges in string theory 12

3.1 Classical string solutions [12

3.2 Bäcklund transformation and the conserved charges 14

3.3 Perturbative matching of string and gauge theory charges 16

3.4 Exact generating function of commuting charges in string theory 19

4. Matching the entire infinite tower of commuting charges 23

A. Exact solution of the Bäcklund equations and generating function 25

A.1 Folded case 25

A.2 Circular case 27

A.3 The Gauss-Landen transformation 30

B. Conventions for elliptic integrals and elliptic functions 31

\section{Introduction and summary}

Recently novel quantitative, dynamical tests of the duality between superconformal $\mathcal{N}=4$ Yang-Mills theory and type IIB superstrings on the $A d S_{5} \times S^{5}$ space-time background have been performed [1]-[5]. They go beyond the BMN and the planewave approximations to, respectively, gauge and string theories [6], and rely on two

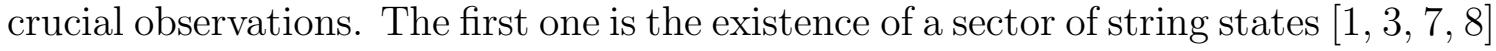
which is accessible by semiclassical [9, 10] methods, i.e. one avoids the unsolved problem of string quantization on a curved background. The second observation is that planar $\mathcal{N}=4$ super Yang-Mills theory is integrable in the one-loop approximation, where it can be mapped to an integrable (super) spin chain [11, 12, 13] ${ }^{1}$. By using

\footnotetext{
${ }^{1}$ It has been conjectured that the integrability extends to all loops [14].
} 
the powerful method of the algebraic Bethe ansatz the latter property allows one to find the exact one-loop anomalous dimensions of arbitrary local composite operators in the gauge theory and compare them with energies of the corresponding dual semiclassical string states.

Specifically, on the string theory side one looks for solitonic solutions of the classical string sigma model with finite energy and spins (i.e. the commuting global charges of the $\mathrm{SO}(4,2) \times \mathrm{SO}(6)$ isometry group of the $A d S_{5} \times S^{5}$ background). With a natural spinning ansatz for the solution the sigma model evolution equations are reduced to that of the Neumann integrable system [4. The latter have solutions of two different types corresponding to so-called folded and circular strings. Quite remarkably, in the thermodynamic limit the Bethe equations encoding the one-loop anomalous dimensions of the gauge theory operators exhibit the same types of solutions [2]. Moreover, one can show that in the limit of large spins ${ }^{2}$ the first subleading (order $\lambda$ ) term in the string energy, which is a transcendental, parametric function of the spins, precisely agrees with the one-loop anomalous dimension of the corresponding operators both for the folded [2, 3] and for the circular [4] configuration type.

It is clear that the Neumann system and the spin chain exhibit very different types of integrability. The first, striking difference is that one compares the classical Neumann system to a quantum spin chain ${ }^{3}$. The second difference is that the Neumann model has a finite number (actually, just two) of integrals of motion while the spin chain exhibits, in the thermodynamical limit, an infinite set of local, commuting integrals of motion. In both systems there are thus precisely as many integrals of motion as required in order to allow for an exact solution. For the Neumann model a certain linear combination of the two integrals gives the space-time energy of the string. The model describes rigid strings (whose shape is time-independent) and the ultimate role of the two integrals appears in a rather mild fashion: they merely specify the topology of the solution (folded or circular). In gauge theory these different string topologies are reflected in the way the Bethe roots are distributed in the complex plane. The energy is given by the lowest charge, but finding it appears to require simultaneously diagonalizing all charges. This is precisely what the Bethe ansatz achieves. But what is the meaning of this infinite set of charges in the framework of the Neumann model? It is clearly important to ask whether one can find them in the dual string theory description as well. Answering this question is the main goal of this paper.

Before we proceed let us mention that the possible interrelation between the integrability of the string theory on the $A d S_{5} \times S^{5}$ background and its field theory

\footnotetext{
${ }^{2}$ Possible connections with the tensionless limit of string theory and gauge theory multiplet shortenings have recently been discussed in 15$]$.

${ }^{3}$ See [16] for some ideas based on relation between the discretized Neumann system and the XYZ spin chain.
} 
counterpart has been recently discussed from the viewpoint of nonabelian (nonlocal) symmetries generated by Yangians [17, 18, 19] (see also [20] for further developments). We feel however that it is of primary importance to first understand the map between the infinite families of local commuting integrals of motion, whose existence is crucial for the solubility of the gauge and string theories at hand.

Actually it is well-known, starting from the work of Pohlmeyer [21], that the classical principle sigma models and their various reductions (in particular the $\mathrm{O}(\mathrm{n})$ vector models we are interested in here) possess an infinite family of local commuting charges [22, 23, 24]. The easiest way, perhaps, to exhibit this family is to use the so-called Bäcklund transformation.

Let us now come to the description of the present work and the results obtained. Our consideration starts from the one-loop gauge theory. We first construct the generating function (resolvent) of the infinite family of commuting local spin chain charges $Q_{n}$, as obtained from the Bethe solutions corresponding to folded and circular string configurations. The first non-trivial charge $Q_{2}$, obtained already previously in [2, 5], is the eigenvalue of the one-loop dilatation operator, i.e. it gives the anomalous dimension. An intriguing feature of the family of gauge theory charges is that, if properly defined, they satisfy "BMN scaling" [6]: The $n$-th charge $Q_{n}$ scales as $J^{1-n}$, where $J$ is the total spin (i.e. the length of the chain).

Next, using the Bäcklund transformation for the $\mathrm{O}(6)$ model, we explicitly work out the first few commuting charges $\mathcal{E}_{n}$ in string theory and further evaluate them on the folded and circular type solutions of the Neumann system. Here the first charge appears to coincide with the Hamiltonian of the Neumann system which, due to the Virasoro constraint, gives the energy of the string. In both the gauge and the string theory computations the charges emerge as rather nontrivial combinations of elliptic integrals governed by the gauge/string modular parameter. In each case the modular parameter is determined by a transcendental relation expressing it as a function of the spins. The string theory calculation comprises the all-loop result for the higher charges. In order to compare with the one-loop gauge theory predictions we develop a perturbative expansion of $\mathcal{E}_{n}$. The obtained "one-loop" charges predicted by string theory do not yet satisfy the above mentioned property of BMN scaling. This is shown to be due to mixing effects, and BMN scaling can be recovered after some natural linear redefinitions $\mathcal{E}_{n} \rightarrow \mathcal{Q}_{n}$. Finally, we demonstrate that a Gauss-Landen modular transformation maps the entire family of commuting charges of string theory onto the one of the gauge theory!

This result nicely extends the correspondence picture: not only the energy of a string state matches the anomalous dimension but also the higher string commuting charges can be matched to those of the gauge theory. Although we have demonstrated this matching on some particular string/gauge solutions it is clear that all this hints at a universal relation between the string sigma model and the spin chain: Semiclassically the string sigma model and the spin chain are the same. The Neu- 
mann solutions and their gauge theory counterparts are just distinguished from the "sea" of all possible solutions by specifying the values of the infinite number of local commuting charges. Further evidence for this universality comes from our finding that the just mentioned linear redefinitions do not depend on the solution type.

The knowledge of the whole family of the string commuting charges relies essentially on on the knowledge of the solution of the Bäcklund equations. As we will see the perturbative solution can be pushed to any desired order in the spectral parameter $\gamma$ but it does not allow us to obtain all the charges; an exact, i.e. nonperturbative in $\gamma$, solution of the Bäcklund equations is needed. Fortunately, the problem of finding an exact solution turns out to have a beautiful resolution. The perturbative treatment shows that the Bäcklund solution still solves the evolution equations of the Neumann model. On the other hand we know the most general solution of the Neumann model; for the two-spin case it depends on one arbitrary constant $\nu$, which can be assumed to depend on the spectral parameter $\gamma$. The Bäcklund equations are then used to determine the function $\nu \equiv \nu(\gamma)$. In this way we get the explicit spectral-dependent solution and use it to obtain the exact generating function (string spectral curve) $\mathcal{E}(\gamma)$ of commuting charges in string theory. The final expression for $\mathcal{E}(\gamma)$ turns out to be rather simple. However, it generates unimproved charges, i.e. the charges do not yet obey BMN scaling. As was already mentioned, the charges with the "proper" one-loop BMN scaling can be obtained from the original unimproved charges by a certain linear redefinition, as we verified explicitly to high order. This does not come as a surprise: Within the commuting family there is always freedom to apply arbitrary linear transformations on the complete set of charges. Using this freedom we are able to derive the complete generating function of higher one-loop gauge theory charges from string theory!

The upshot of the present work is then that we shed light on matching the integrable structures on the gauge and string sides of the AdS/CFT correspondence. This is done by way of example, for two specific cases. It would be very important to find the underlying general reasons for our findings. The structures on the gauge and string sides are still conceptually rather distinct. E.g. on the gauge side the commuting charges are actually a crucial device, as they appear to be needed to find the energies; on the other hand, on the string side we can find the energies without requiring the knowledge of the higher charges: here they emerge, at this point, as a mere add-on. The reason for that can be traced to our assumption of the Neumann (rotating) ansatz which considerably restricts the possible string trajectories. To end, we quite generally feel that the local commuting charges might well hold the key to unraveling the mysterious integrable structures of the planar AdS/CFT system.

The paper is organized as follows. In chapter 2 we explain how to derive the generating function of commuting charges in one-loop gauge theory, first quite generally, and subsequently in the specific case of the "folded" and "circular" root distribu- 
tions. This is done by inspecting the detailed statistical distribution of Bethe roots in the integrable quantum spin chain approach. In chapter 3 we first discuss the classical string solutions within the Neumann integrable ansatz. Then we describe a perturbative construction of the Bäcklund transformations and subsequently use it to compute the first few commuting charges in string theory. We furthermore show that in the one-loop approximation these charges can be precisely matched to those in the gauge theory. Then we show how the perturbative construction of the Bäcklund transformation allows one to guess an ansatz for an exact solution of the Bäcklund equations. In appendix A the Bäcklund equations are solved exactly and the solution is used in chapter 3 to construct the complete generating function for commuting charges in string theory. As mentioned above this function generates unimproved charges. We therefore demonstrate in the final chapter 4 how to extract the complete infinite set of one-loop gauge charges from the string generating function. To keep the presentation more transparent, we restrict ourselves in the main text devoted to the string charges to the case of the folded string; the circular string is treated separately in appendix A. In appendix A we also discuss in some detail the modular Gauss-Landen transformation which relates the gauge and the string theory quantities and explain its geometric meaning in our present context. In appendix B our conventions for elliptic integrals and elliptic functions are summarized.

\section{Higher one-loop charges in gauge theory}

\subsection{Generalities}

Let us continue the study of the spectrum of the following single trace $\mathcal{N}=4$ gauge theory operators which were shown to be dual to $A d S$ strings rotating on the five sphere $S^{5}$ in two orthogonal planes with large angular momenta $J_{1}, J_{2}$ [2, 3, 5]:

$$
\operatorname{Tr} Z^{J_{1}} \Phi^{J_{2}}+\ldots
$$

These operators are composites of two $(Z, \Phi)$ out of the three complex scalar fields of the $\mathcal{N}=4$ gauge theory. The dots in eq.(2.1) indicate that the operator mixes in the $N=\infty$ planar gauge theory with all single trace operators containing arbitrary sequences of $J_{1} Z$ and $J_{2} \Phi$ fields. We therefore need to diagonalize, i.e. find the eigensystem, in the space of all these operators. This is a formidable problem even at the one-loop level; it is solved by mapping it to the spectral problem of an equivalent XXX Heisenberg quantum spin chain [11], where the two fields are interpreted as, respectively, up-spins and down-spins. The exact planar one-loop spectrum $\frac{1}{4 \pi^{2}} Q_{2}$ of these operators is then parametrically determined [11] by the expression

$$
Q_{2}=\frac{J}{2} \sum_{j=1}^{J_{2}} \frac{1}{u_{j}^{2}+1 / 4}
$$


where the parameters $u_{j}$ (the Bethe roots) are found by solving the following set of Bethe equations for all $J_{1} \geq J_{2}$, where $J=J_{1}+J_{2}$ :

$$
\left(\frac{u_{j}+i / 2}{u_{j}-i / 2}\right)^{J}=\prod_{\substack{k=1 \\ k \neq j}}^{J_{2}} \frac{u_{j}-u_{k}+i}{u_{j}-u_{k}-i} \quad \prod_{j=1}^{J_{2}} \frac{u_{j}+i / 2}{u_{j}-i / 2}=1 .
$$

No two roots are allowed to coincide. A similar but obviously more involved situation applies to arbitrary composite operators in $\mathcal{N}=4$ gauge theory, where the Bethe equations are also known [13].

We are interested in the thermodynamic limit $J, J_{2} \rightarrow \infty$ of these Bethe equations with

$$
\alpha \equiv \frac{J_{2}}{J}
$$

held fixed. The Bethe roots are expected to condense onto a union $\mathcal{C}=\cup_{n} \mathcal{C}_{n}$ of smooth contours $\mathcal{C}_{n}$ in the complex plane of the continuous variable $u$ :

$$
\frac{u_{j}}{J} \rightarrow u
$$

Their distribution may be described by a density $\rho(u)$ having support $u \in \mathcal{C}$ on the union of contours:

$$
\rho(u)=\frac{1}{J} \sum_{j=1}^{J_{2}} \delta\left(u-\frac{u_{j}}{J}\right), \quad \text { hence } \quad \int_{\mathcal{C}} d u \rho(u)=\alpha .
$$

The density allows to find the energy eq.(2.2) in the thermodynamic limit.

$$
Q_{2}=\frac{1}{2} \int_{\mathcal{C}} d u \frac{\rho(u)}{u^{2}}
$$

The discrete Bethe equations eqs.(2.3) turn into

$$
f_{\mathcal{C}} d v \frac{\rho(v) u}{v-u}=\frac{1}{2}+\pi n_{\mathcal{C}(u)} u, \quad \text { where } \quad u \in \mathcal{C}_{n} \quad \text { and } \quad \int_{\mathcal{C}} d u \frac{\rho(u)}{u}=0 .
$$

This system of singular integral equations (the bar through the integral indicates a principal part prescription) determines the density. The mode numbers $n_{\mathcal{C}(u)}$ are integers which are expected to be constant on each smooth component $\mathcal{C}_{n}$ of the density support $\mathcal{C}$. They appear since we need to take a logarithm of the discrete equations (2.3) in order to derive eqs.(2.8), and are subject to the condition $\int_{\mathcal{C}} d u \rho(u) n_{\mathcal{C}(u)}=0$.

The solutions of the system of equations eqs.(2.8) have not yet been studied in any generality. Such a study would presumably lead to a classification of the large $J_{1}, J_{2}$ spectrum of the operators (2.1). In general we expect solutions for any number of cuts $\mathcal{C}_{i}$. An even larger system of singular integral equations, which has not even yet been written down in public, governs the full set of Bethe equations [13] for arbitrary single trace operators in the thermodynamic limit. What has been 
done was a study of the simplest kinds of solution of eqs. 2.8) involving one or two contours [2, 5]. These solutions are reviewed and further analyzed in sections 2.2. and 2.3 .

At this point we should realize that the above solution of the "thermodynamic" spectral problem of the operators eq.(2.1) contains a lot more information than we have used so far. The Bethe integral equations eqs.(2.8) allow to determine the root distribution density $\rho(u)$ which is then merely used to extract the energy eigenvalue eq. (2.7).

E.g. the so far obtained solutions [2, 5] found for $\rho(u)$ an elliptic curve, ubiquitously present in integrable systems. The resulting charge $Q_{2}$ was successfully matched to a string computation [3, 5]. It is thus very natural to ask whether the entire "gauge curve" $\rho(u)$ also has an interpretation on the string side. We will find that the answer to this question is: Yes indeed! In fact, solving the Bethe ansatz equations eqs.(2.3) leads not only to the diagonalization of the Hamiltonian $Q_{2}$ (=lowest charge) of the spin chain, but also simultaneously diagonalizes the entire set of commuting charges $Q_{k}$. These are generated by the transfer matrix $T(u)$ of the discrete spin chain through

$$
Q_{k+1}=-\left.\frac{i}{2} \frac{J^{k}}{k !} \frac{d^{k}}{d u^{k}} \log T(u)\right|_{u=\frac{i}{2}}
$$

Each Bethe state, determined through eqs.(2.3), is an eigenstate of $T(u)$ for arbitrary complex values of the spectral parameter $u$. Its eigenvalue is

$$
T(u)=\left(u+\frac{i}{2}\right)^{J} \prod_{j=1}^{J_{2}} \frac{u-u_{j}-i}{u-u_{j}}+\left(u-\frac{i}{2}\right)^{J} \prod_{j=1}^{J_{2}} \frac{u-u_{j}+i}{u-u_{j}} .
$$

Dropping an irrelevant $u_{j}$-independent constant, we find in the thermodynamic limit (2.5), (2.6) for the charges

$$
Q_{k}=\frac{1}{2} \int_{\mathcal{C}} d u \frac{\rho(u)}{u^{k}}
$$

Clearly the commuting charges are non-unique up to linear (or even polynomial) redefinitions. Our definition eq.(2.9), which is standard except for the factor $J^{k}$, ensures that the charges behave properly (i.e. they are of order $\mathcal{O}(1)$ ) in the scaling limit (2.5), 2.6). It is natural to introduce a generating function of all conserved charges $Q_{k}$ (the resolvent). It is given by

$$
H(u)=\frac{\alpha}{2}+\frac{1}{2} \sum_{k=1}^{\infty} Q_{k} u^{k}, \quad \text { i.e. } \quad H(u)=\frac{1}{2} \int_{\mathcal{C}} d v \rho(v) \frac{v}{v-u}
$$

and thus analytically defined throughout the complex $u$ plane, except for the cuts $\mathcal{C}_{i}$. Let us now, for the reminder of this chapter, compute the resolvent for the known cases of the "folded" and "circular" cases and thereby derive the infinite number of commuting charges for these solutions. In the next chapter these one-loop charges will be reproduced on the string side. 


\subsection{Folded case}

Let us first reconsider the large $J_{1}, J_{2}$ ground state energy of the operators (2.1) [2, 5]. In this case the roots condense on two curved, convex cuts $\mathcal{C}=\mathcal{C}^{+} \cup \mathcal{C}^{-}$ which are symmetric w.r.t reflection on both the real and imaginary axis. In order to have explicit formulas, we will again discuss the related case where the two cuts $\left(\mathcal{C}^{-} \rightarrow[-b,-a]\right.$ and $\left.\mathcal{C}^{+} \rightarrow[a, b]\right)$ are located on the real axis in the $u$-plane: This corresponds to analytically continuing the filling fraction $\alpha=J_{2} / J$ to negative values [2, 司]. The thermodynamic Bethe equations become

$$
f_{a}^{b} d v \frac{\rho(v) u^{2}}{v^{2}-u^{2}}=\frac{1}{4}-\frac{\pi}{2} u, \quad Q_{2}=\int_{a}^{b} d u \frac{\rho(u)}{u^{2}} .
$$

The first equation is closely related to the saddle point equation of the $\mathrm{O}( \pm 2)$ matrix model (see e.g. 25]). Physically this corresponds to a string rotating with one spin on $A d S_{5}$ and one angular momentum on $S^{5}$ [5], but we will not make use of this fact in this paper. The previously obtained solution reads

$$
\rho(u)=\frac{2}{\pi u} f_{a}^{b} d v \frac{v^{2}}{v^{2}-u^{2}} \sqrt{\frac{\left(b^{2}-u^{2}\right)\left(u^{2}-a^{2}\right)}{\left(b^{2}-v^{2}\right)\left(v^{2}-a^{2}\right)}} .
$$

with the normalization (remember $\alpha<0$, i.e. formally $J<0$ )

$$
\int_{a}^{b} d u \rho(u)=-\frac{\alpha}{2}
$$

The interval boundaries are determined by

$$
a=\frac{1}{4 \mathrm{~K}(q)}, \quad b=\frac{1}{4 \sqrt{1-q} \mathrm{~K}(q)} .
$$

Here $\mathrm{K}(q)$ is the complete elliptic integral of the first kind (see appendix B on our conventions for elliptic integrals and elliptic functions). The density may be expressed explicitly through the elliptic integral of the third kind:

$$
\rho(u)=\frac{1}{2 \pi b u} \sqrt{\frac{u^{2}-a^{2}}{b^{2}-u^{2}}}\left[\frac{b^{2}}{a}-4 u^{2} \Pi\left(\frac{b^{2}-u^{2}}{b^{2}}, q\right)\right], \quad q=\frac{b^{2}-a^{2}}{b^{2}},
$$

From this it is straightforward to obtain the energy eigenvalue $Q_{2}$ in the parametric form

$$
Q_{2}=2 \mathrm{~K}(q)[(2-q) \mathrm{K}(q)-2 \mathrm{E}(q)]
$$

with

$$
-\alpha \equiv-\frac{J_{2}}{J}=\frac{1}{2 \sqrt{1-q}} \frac{\mathrm{E}(q)}{\mathrm{K}(q)}-\frac{1}{2} .
$$

This rather involved final result, first obtained in [2, 5] agrees with the one-loop prediction of semiclassical string theory [3, 5]. This ends our short review and we 
will now compute the higher charges, using the procedure explained in the previous section.

For the even charges (the odd charges vanish on the unpaired ground state we are interested in) we have

$$
\begin{gathered}
Q_{2 k}=\int_{a}^{b} d u \frac{\rho(u)}{u^{2 k}} . \\
H(u)=-\frac{\alpha}{2}+\sum_{k=1}^{\infty} Q_{2 k} u^{2 k}, \quad \text { i.e. } \quad H(u)=\int_{a}^{b} d v \rho(v) \frac{v^{2}}{v^{2}-u^{2}} .
\end{gathered}
$$

On the cut $u \in[a, b]$ this function behaves as $H(u \pm i \epsilon)=-\frac{\alpha}{2}+\frac{1}{4}-\frac{\pi}{2} u \pm i \frac{\pi}{2} u \rho(u)$. It is therefore straightforward to obtain an integral representation of the resolvent

$$
H(u)=-\frac{\alpha}{2}+\frac{1}{4}-\int_{a}^{b} \frac{d v v^{2}}{v^{2}-u^{2}} \sqrt{\frac{\left(b^{2}-u^{2}\right)\left(a^{2}-u^{2}\right)}{\left(b^{2}-v^{2}\right)\left(v^{2}-a^{2}\right)}}
$$

Just like the density eq. 2.17) we can also express it explicitly with the help of the elliptic integral of the third kind:

$$
H(u)=-\frac{\alpha}{2}+\frac{1}{4}-\frac{\pi}{2} u-\frac{1}{4 b} \sqrt{\frac{a^{2}-u^{2}}{b^{2}-u^{2}}}\left[\frac{b^{2}}{a}-4 u^{2} \Pi\left(\frac{b^{2}-u^{2}}{b^{2}}, q\right)\right] .
$$

This is not the most convenient form to perform the power series expansion around $u=0$. A further, more suitable representation for $H(u)$ is

$$
H(u)=-\frac{\alpha}{2}+\frac{1}{4}-\frac{a^{2}}{b} \sqrt{\frac{b^{2}-u^{2}}{a^{2}-u^{2}}} \Pi\left(-q \frac{u^{2}}{a^{2}-u^{2}}, q\right) .
$$

From this expression we can easily obtain the explicit form of the higher charges; the first few read

$$
\begin{aligned}
Q_{4}= & \frac{2^{3}}{3} \mathrm{~K}(q)^{3}\left[4(q-2) \mathrm{E}(q)+\left(8-8 q+3 q^{2}\right) \mathrm{K}(q)\right] \\
Q_{6}= & \frac{2^{6}}{5} \mathrm{~K}(q)^{5}\left[-2\left(8-8 q+3 q^{2}\right) \mathrm{E}(q)+\left(16-24 q+18 q^{2}-5 q^{3}\right) \mathrm{K}(q)\right] \\
Q_{8}= & \frac{2^{7}}{7} \mathrm{~K}(q)^{7}\left[8(q-2)\left(8-8 q+5 q^{2}\right) \mathrm{E}(q)+\left(128-256 q+288 q^{2}-160 q^{3}+35 q^{4}\right) \mathrm{K}(q)\right] \\
Q_{10}= & \frac{2^{10}}{9} \mathrm{~K}(q)^{9}\left[\left(-256+512 q-576 q^{2}+320 q^{3}-70 q^{4}\right) \mathrm{E}(q)\right. \\
& \left.\quad-(q-2)\left(128-256 q+352 q^{2}-224 q^{3}+63 q^{4}\right) \mathrm{K}(q)\right]
\end{aligned}
$$

In the next chapter we will demonstrate how to "perturbatively" (in $u$ ) find these charges on the string side. In the final chapter 4 we will then rigorously derive the entire generating function eq.(2.24) from string theory. However, before doing so we will also treat the Bethe solution corresponding to the circular string. 


\subsection{Circular case}

The configuration of Bethe roots corresponding to the circular string was found in [2], chapter 4 (see also [5], appendix D). As opposed to the folded case, this is not the ground state of the spin chain. For the convenience of the reader we will closely follow $^{4}$ [2, 5]. In the present case the roots are distributed on a single, connected curve $\mathcal{C}$ along the imaginary axis. Around the origin an odd number $J_{2}^{\prime}$ (with $J_{2}^{\prime}<J_{2} \leq J_{1}$ and $J=J_{1}+J_{2}$ ) of roots are placed in the near vicinity of the half-integer purely imaginary values, i.e. their density is constant $(=2$ since there are two roots per integer interval on the imaginary axis), up to $\pm i c$ with $c=\frac{J_{2}^{\prime}-1}{4 J}$. Further away the distribution is less dense (i.e. $\widetilde{\sigma}(x)<2$ ) and non-constant. Let us replace the variable $u$ parametrizing the Bethe plane by $u=i x$. The density $\sigma(x) \equiv \rho(u)$ then has the form

$$
\sigma(x)=\frac{1}{J} \sum_{j=1}^{J_{2}} \delta\left(x+i \frac{u_{j}}{J}\right) \rightarrow \sigma(x)= \begin{cases}2 & -c<x<c \\ \tilde{\sigma}(x) & c<x<d \\ \tilde{\sigma}(-x) & -d<x<-c \\ 0 & x<-d \text { or } d<x\end{cases}
$$

Due to the constant condensate around the origin we again end up with a two-cut problem, despite the fact that all roots lie on one continuous curve $\mathcal{C}$ ! The continuum Bethe equations and, respectively, the lowest charge (i.e. energy) read

$$
f_{c}^{d} d y \tilde{\sigma}(y) \frac{x^{2}}{x^{2}-y^{2}}=\frac{1}{4}-x \log \frac{x+c}{x-c} \quad Q_{2}=\frac{2}{c}-\int_{c}^{d} d x \frac{\tilde{\sigma}(x)}{x^{2}} .
$$

The solution is

$$
\tilde{\sigma}(x)=\frac{1}{2 \pi} \sqrt{\left(d^{2}-x^{2}\right)\left(x^{2}-c^{2}\right)}\left(-\frac{1}{x c d}+4 \int_{-c}^{c} \frac{d y}{x-y} \frac{1}{\sqrt{\left(d^{2}-y^{2}\right)\left(c^{2}-y^{2}\right)}}\right),
$$

with the normalization

$$
2 c+\int_{c}^{d} d x \widetilde{\sigma}(x)=\frac{\alpha}{2}
$$

The interval boundaries are determined through

$$
c=\frac{1}{8 \mathrm{~K}(r)}, \quad d=\frac{1}{8 \sqrt{r} \mathrm{~K}(r)} .
$$

The density may again be expressed explicitly

$$
\widetilde{\sigma}(x)=2-\frac{4}{\pi d x} \sqrt{\left(d^{2}-x^{2}\right)\left(x^{2}-c^{2}\right)} \Pi\left(\frac{x^{2}}{d^{2}}, r\right), \quad r=\frac{c^{2}}{d^{2}} .
$$

\footnotetext{
${ }^{4}$ However, in order (1) to avoid confusions with the notation in other parts of the present paper, and (2) to agree with the perhaps more natural normalization of the folded string, we have decided to make the following notational changes w.r.t. [2, 5]: (1) $q \rightarrow x, q^{\prime} \rightarrow y, s \rightarrow c, t \rightarrow d$ and (2) $\sigma(q) \rightarrow 2 \sigma(x)$.
} 
The energy eigenvalue is, similar to, but different from eq.(2.18), parametrically given by

$$
Q_{2}=8 \mathrm{~K}(r)[2 \mathrm{E}(r)+(r-1) \mathrm{K}(r)]
$$

with

$$
\alpha \equiv \frac{J_{2}}{J}=\frac{\sqrt{r}-1}{2 \sqrt{r}}+\frac{1}{2 \sqrt{r}} \frac{\mathrm{E}(r)}{\mathrm{K}(r)} .
$$

These are the gauge results derived for the circular string in [2, 5] and successfully compared to semiclassical string theory in [4].

As for the previous case of the folded solution we would now like to also extract the higher charges from the density function eq.(2.31). Naively these should be given, cf eqs.(2.11), (2.20), by the expression

$$
Q_{2 k} \stackrel{?}{=}(-1)^{k} \int_{0}^{d} d x \frac{\sigma(x)}{x^{2 k}}
$$

However, this formula is clearly nonsensical: The density $\sigma(x)$ is constant at the origin, and the above expression therefore diverges at $x=0$. Let us go back to the original definition of the higher charges for the finite spin chain with discrete roots $u_{j}=i x_{j}$, i.e. eqs.(2.9),(2.10), and investigate the thermodynamic limit more carefully. As we are assuming $J$ to be large (i.e. the second term in the transfer matrix eq.(2.10) does not contribute) we have

$$
Q_{2 k}=\frac{i^{2 k}}{2} \frac{J^{2 k-1}}{(2 k-1) !}\left[\frac{d^{2 k-1}}{d x^{2 k-1}} \log \prod_{j=1}^{J_{2}} \frac{x-x_{j}-1}{x-x_{j}}\right]_{x=\frac{1}{2}}+\ldots
$$

where the dots indicate an $x_{j}$-independent constant, which we can drop. We had assumed that all roots $u_{j}=i x_{j}$ scale as $\sim J$. This is clearly not the case for the roots near the origin. However, these are condensed at half-integer values of the imaginary axis, and their contribution to the transfer matrix mostly cancels, see eq.(2.35). We therefore have

$$
Q_{2 k}=\frac{i^{2 k}}{2} \frac{J^{2 k-1}}{(2 k-1) !}\left[\frac{d^{2 k-1}}{d x^{2 k-1}}\left(\log \frac{\left(x-\frac{J_{2}^{\prime}+3}{4}\right)\left(x-\frac{J_{2}^{\prime}+1}{4}\right)}{\left(x+\frac{J_{2}^{\prime}-3}{4}\right)\left(x+\frac{J_{2}^{\prime}-1}{4}\right)}+\log \prod_{j} \frac{x-\tilde{x}_{j}-1}{x-\tilde{x}_{j}}\right)\right]_{x=\frac{1}{2}}
$$

where the last product runs only over the $J_{2}-J_{2}^{\prime}$ roots $\tilde{x}_{j}$ located outside the condensate, and distributed according to the density $\tilde{\sigma}(x)$. Taking the thermodynamic limit $J, J_{2}, J_{2}^{\prime} \rightarrow \infty$ we find that the charges are actually perfectly finite and given by

$$
Q_{2 k}=\frac{2}{2 k-1} \frac{1}{c^{2 k-1}}-\int_{c}^{d} d x \frac{\tilde{\sigma}(x)}{x^{2 k}} .
$$

(For esthetic reasons we have dropped an overall immaterial sign $(-1)^{k+1}$ in this expression.) Eq.(2.37) generalizes a result proven by different means in [2] for the 
energy $\left(Q_{2}\right)$ to arbitrary $2 k$. Interestingly, eq.(2.37) coincides with an ad-hoc regularization of the naive formula eq.(2.34), where one just drops the divergence at the lower limit of the integration. The generating function of the charges eq.(2.37) (i.e. the resolvent) is found to be

$$
H(x)=\frac{\alpha}{2}-\sum_{k=1}^{\infty} Q_{2 k} x^{2 k} \quad \text { i.e. } \quad H(x)=2 c+x \log \frac{c-x}{c+x}+\int_{c}^{d} d y \widetilde{\sigma}(y) \frac{y^{2}}{y^{2}-x^{2}}
$$

We can again express it explicitly with the help of the elliptic integral of the third kind:

$$
H(x)=\frac{\alpha}{2}-\frac{1}{4}+\frac{2}{d} \sqrt{\left(d^{2}-x^{2}\right)\left(c^{2}-x^{2}\right)} \Pi\left(\frac{x^{2}}{d^{2}}, r\right) .
$$

From these expressions we can easily obtain the explicit form of the higher charges; the first few are

$$
\begin{aligned}
Q_{4}= & \frac{2^{7}}{3} \mathrm{~K}(r)^{3}[4(1+r) \mathrm{E}(r)+(r-1)(3 r+1) \mathrm{K}(r)] \\
Q_{6}= & \frac{2^{12}}{5} \mathrm{~K}(r)^{5}\left[\left(6+4 r+6 r^{2}\right) \mathrm{E}(r)+(r-1)\left(1+2 r+5 r^{2}\right) \mathrm{K}(r)\right], \\
Q_{8}= & \frac{2^{15}}{7} \mathrm{~K}(r)^{7}\left[8(r+1)\left(5-2 r+5 r^{2}\right) \mathrm{E}(r)+(r-1)\left(5+9 r+15 r^{2}+35 r^{3}\right) \mathrm{K}(r)\right], \\
Q_{10}= & \frac{2^{20}}{9} \mathrm{~K}(r)^{9}\left[\left(70+40 r+36 r^{2}+40 r^{3}+70 r^{4}\right) \mathrm{E}(r)\right. \\
& \left.+(r-1)\left(7+12 r+18 r^{2}+28 r^{3}+63 r^{4}\right) \mathrm{K}(r)\right],
\end{aligned}
$$

Using the methods that will be exposed in the next two chapters, we will derive in appendix A.2 the charges (2.40), and actually the entire generating function eq. (2.39) from string theory.

\section{Higher semiclassical charges in string theory}

\subsection{Classical string solutions}

The classical closed bosonic string propagating in the $A d S_{5} \times S^{5}$ space-time is described in terms of the $\mathrm{O}(6) \times \mathrm{O}(4,2)$ sigma model. As was shown in [4], rigid string solitons (i.e. strings with a time-independent profile) are classified in terms of periodic solutions of the Neumann integrable system. The Neumann system arises from the sigma model equations of motion after specifying a rotating string ansatz for the embedding coordinates. ${ }^{5}$ In particular, for the motion on $S^{5}$ the rotating ansatz

\footnotetext{
${ }^{5}$ More precisely, one obtains two Neumann systems: The "compact" system originating from the $\mathrm{O}(6)$ part and the "non-compact" one related to the $\mathrm{O}(4,2)$ part. The conformal gauge constraint imposes one equation between the conserved energies of the systems.
} 
reads

$$
X_{1}+i X_{2}=x_{1}(\sigma) e^{i w_{1} \tau}, \quad X_{3}+i X_{4}=x_{2}(\sigma) e^{i w_{2} \tau}, \quad X_{5}+i X_{6}=x_{3}(\sigma) e^{i w_{3} \tau},
$$

where $X_{i}$ are the embedding coordinates of $S^{5}$. The variables $x_{i}(\sigma)$ satisfy the dynamical equations of the Neumann system and the relation

$$
\sum_{i=1}^{3} x_{i}^{2}=1
$$

which implies that at a fixed moment in time the string lies on a two-dimensional sphere $S^{2}$. In addition the $x_{i}(\sigma)$ obey the closed string periodicity condition:

$$
x_{i}(\sigma+2 \pi)=x_{i}(\sigma) .
$$

The rotation on $S^{5}$ with the frequencies $w_{i}$ gives rise to three angular momentum integrals (spins) $J_{i}=\sqrt{\lambda} \mathcal{J}_{i}$. Due to the Virasoro constraint the space-time energy of the string $E=\sqrt{\lambda} \mathcal{E}$ becomes a function of the spins:

$$
E=E\left(J_{1}, J_{2}, J_{3}\right)
$$

From now on we will restrict our consideration to string solitons carrying only two non-vanishing spin components, $J_{1}$ and $J_{2}$. Needless to say, it would be quite interesting to extend the analysis below to the general three-spin case, using as an input [四.

Recall that the solutions for $x_{i}$ depend on a discrete choice for the relative position of the two Neumann integrals w.r.t. to the frequencies $w_{i}^{2}$ : these can be of two types, corresponding to folded or circular string geometries. Below we will review these solutions [3], 4].

\section{Folded string solution}

The embedding coordinates for the folded string configuration are solved in terms of the standard Jacobi elliptic functions (cf. appendix B) as follows $\left(w_{i j}^{2} \equiv w_{i}^{2}-w_{j}^{2}\right)$ :

$$
x_{1}(\sigma)=\operatorname{dn}\left(\sigma \sqrt{w_{21}^{2}}, t\right), \quad x_{2}(\sigma)=\sqrt{t} \operatorname{sn}\left(\sigma \sqrt{w_{21}^{2}}, t\right), \quad x_{3}(\sigma)=0,
$$

Here the Jacobi modulus $t$ is determined through the closed string periodicity condition (for simplicity we consider the solution with the winding number $n=1$, cf. the general discussion of winding numbers in [4]):

$$
\frac{\pi}{2} \sqrt{w_{21}^{2}}=\mathrm{K}(t), \quad w_{2}>w_{1}
$$

and, solving for w's in terms of spins $\mathcal{J}_{i}$, it can be further found from the equation

$$
\left(\frac{\mathcal{J}_{2}}{\mathrm{~K}(t)-\mathrm{E}(t)}\right)^{2}-\left(\frac{\mathcal{J}_{1}}{\mathrm{E}(t)}\right)^{2}=\frac{4}{\pi^{2}} .
$$




\section{Circular string solution}

The circular type configuration is described by

$$
x_{1}(\sigma)=\operatorname{sn}\left(\sigma \sqrt{\frac{w_{12}^{2}}{t}}, t\right), \quad x_{2}(\sigma)=\operatorname{cn}\left(\sigma \sqrt{\frac{w_{12}^{2}}{t}}, t\right), \quad x_{3}(\sigma)=0,
$$

while the equation for the modulus (again for the winding number $n=1$ ) is

$$
\frac{\pi}{2} \sqrt{\frac{w_{12}^{2}}{t}}=\mathrm{K}(t), \quad w_{1}>w_{2}
$$

and it can be reexpressed via the spins as follows

$$
\left(\frac{\mathcal{J}_{1}}{\mathrm{~K}(t)-\mathrm{E}(t)}\right)^{2}-\left(\frac{\mathcal{J}_{2}}{(1-t) \mathrm{K}(t)-\mathrm{E}(t)}\right)^{2}=\frac{4}{\pi^{2} t} .
$$

In fact, for the case of two non-zero spins the evolution equations of the Neumann model coincide with those of a plane pendulum in a gravitational field. Its motion has two phases, either oscillatory or rotating, which in the present stringy context translate into the folded or circular type solutions, respectively.

\subsection{Bäcklund transformation and the conserved charges}

One possible way to construct an infinite family of local commuting integrals of motion for a sigma model is to use the Bäcklund transformations. These transform a solution of the evolution equations into a new one. The transformation can be usually constructed by perturbing ("dressing") some trial solution. ${ }^{6}$

Recall that in light-cone coordinates $\xi, \eta$, where $\tau=\xi+\eta$, and $\sigma=\xi-\eta$, the evolution equations for the $\mathrm{O}(6)$ sigma model read as

$$
X_{\xi \eta}+\left(X_{\xi} \cdot X_{\eta}\right) X=0, \quad(X \cdot X)=1
$$

where $X_{\xi}$ denotes the derivative of $X$ w.r.t. to the variable $\xi$, and so on, and $\left(X_{\xi} \cdot X_{\eta}\right)$ is the scalar product of two vectors.

Our basic idea is to use the folded or circular strings as trial solutions and to dress them. When the new solution is found it can be used to obtain the values of the local commuting charges on the trial solution.

In terms of the embedding coordinates $X_{i}$ the Bäcklund transformations for the $\mathrm{O}(6)$ model can be described by the following set of equations (see e.g. [23])

$$
\begin{aligned}
2 \gamma^{2}(X(\gamma)+X)_{\xi} & =\left(1+\gamma^{2}\right)\left(X(\gamma) \cdot X_{\xi}\right)(X(\gamma)-X), \\
2(X(\gamma)-X)_{\eta} & =-\left(1+\gamma^{2}\right)\left(X(\gamma) \cdot X_{\eta}\right)(X(\gamma)+X)
\end{aligned}
$$

\footnotetext{
${ }^{6}$ The Bäcklund transformations should not be confused with the so-called dressing transformations whose generators are subject to a nonabelian algebra (see e.g. [26]).
} 
together with the normalization conditions

$$
X(0)=X, \quad(X(\gamma) \cdot X(\gamma))=1, \quad(X(\gamma) \cdot X)=\frac{1-\gamma^{2}}{1+\gamma^{2}} .
$$

Here $\gamma$ is a spectral parameter and $X$ is a trial solution. Solving eqs.(3.11) we obtain another solution $X(\gamma)$ of the evolution equations (3.10) which is the dressing of our trial solution $X$. The equations (3.11) admit a solution in terms of a power series in the spectral parameter $\gamma$

$$
X(\gamma)=\sum_{k=0}^{\infty} X^{(k)} \gamma^{k}
$$

where the coefficients $X^{(k)}$ can be found recurrently to any desired order.

The generating function of the local commuting charges of the $\mathrm{O}(6)$ sigma model

$$
\mathcal{E}(\gamma)=\sum_{k=2}^{\infty} \mathcal{E}_{k} \gamma^{k}
$$

can be obtained from the dressed solution:

$$
\mathcal{E}(\gamma)=\int \frac{d \sigma}{4 \pi}\left[\gamma\left(X(\gamma) \cdot X_{\xi}\right)+\gamma^{3}\left(X(\gamma) \cdot X_{\eta}\right)\right] .
$$

Let us now discuss the perturbative construction of the dressed solution $X(\gamma)$. Plugging the ansatz (3.13) into eqs.(3.11), (3.12) it is easy to determine the first few coefficients $X^{(k)}$ :

$$
X^{(0)}=X, \quad X^{(1)}=\frac{2 X_{\xi}}{\left\|X_{\xi}\right\|}, \quad X^{(2)}=\frac{X_{\xi}^{(1)}}{\left\|X_{\xi}\right\|},
$$

where we have introduced $\left\|X_{\xi}\right\|=\sqrt{\left(X_{\xi} \cdot X_{\xi}\right)}$. The general coefficient $X^{(k)}$ can be obtained recurrently from $X^{(l)}$ with $l<k$ :

$$
X^{(k+1)}=\frac{1}{2\left\|X_{\xi}\right\|}\left[Y^{(k)}-\frac{X_{\xi}}{2\left\|X_{\xi}\right\|^{2}}\left(Y^{(k)} \cdot X_{\xi}\right)\right],
$$

where

$$
Y^{(k)}=2 X_{\xi}^{(k)}-\sum_{l=1}^{k-1}\left[\left(X^{(l)} \cdot X_{\xi}\right) X^{(k-l)}+\left(X^{(l+1)} \cdot X_{\xi}\right) X^{(k+1-l)}\right] .
$$

The coefficients $\mathcal{E}_{k}$ of the generating function $\mathcal{E}(\gamma)$, which are mutually commuting conserved charges, are then given by

$$
\mathcal{E}_{k}=\int \frac{d \sigma}{4 \pi}\left[\left(X^{(k-1)} \cdot X_{\xi}\right)+\left(X^{(k-3)} \cdot X_{\eta}\right)\right], \quad k \geq 2 .
$$

Here $\left(X^{(k)} \cdot X_{-}\right)=0$ for $k$ negative. 


\subsection{Perturbative matching of string and gauge theory charges}

Here we compute the values of the first few conserved charges (3.19) on the solution of the Neumann system corresponding to the folded string and show that up to some natural linear redefinition they match precisely those found in one-loop gauge theory. Matching of the charges on the circular type solution is discussed in appendix A.

The charge $\mathcal{E}_{2}$ follows easily upon substituting the Neumann ansatz into (3.19):

$$
\mathcal{E}_{2}=\int \frac{d \sigma}{4 \pi}\left(X^{(1)} \cdot X_{\xi}\right)=\int \frac{d \sigma}{2 \pi}\left\|X_{\xi}\right\|=\sqrt{2 H} .
$$

Here (the prime denotes a derivative w.r.t. $\sigma$ )

$$
H=\frac{1}{2} \sum_{i=1}^{3}{x_{i}^{\prime 2}}^{2}+w_{i}^{2} x_{i}^{2}
$$

is nothing but the Hamiltonian of the Neumann system. Due to the Virasoro constraint the charge $\mathcal{E}_{2}$ coincides with the space-time energy $\mathcal{E}_{2} \equiv \mathcal{E}$ of the string. Note that $H$ is in fact a "doubly" conserved quantity: It is independent with respect to both $\tau$ and $\sigma$.

The next odd integral $\mathcal{E}_{3}$ is found to be zero as a consequence of the relation $\left(X_{\xi}^{(1)} \cdot X_{\xi}\right)=0$.

In principle, the higher charges, e.g. the fourth one,

$$
\mathcal{E}_{4}=\int \frac{d \sigma}{4 \pi}\left[\left(X^{(3)} \cdot X_{\xi}\right)+\left(X^{(1)} \cdot X_{\eta}\right)\right]
$$

when computed on the generic Neumann solution $x_{i}(\sigma)$, can also be expressed as functionals of $x_{i}$ and its derivative ${ }^{7} x_{i}^{\prime}$, i.e. $\mathcal{E}_{n}=\mathcal{E}_{n}\left(x_{i}, x_{i}^{\prime}\right)$. However, already for $\mathcal{E}_{4}$ the resulting answer is rather complicated and not very instructive. Hence, we restrict ourselves to finding the value of the charges on the special folded string solution described in the previous section.

On the folded string solution (3.4) the result of the evaluation of the integral $\mathcal{E}_{4}$ can be compactly written in the form

$$
\mathcal{E}_{4}=-\frac{16}{\pi^{2} \mathcal{E}_{2}} \mathrm{~K}(t)[\mathrm{E}(t)+(t-1) \mathrm{K}(t)]+\frac{32}{\pi^{4} \mathcal{E}_{2}^{3}} t(t-1) \mathrm{K}(t)^{4} .
$$

Some comments are in order. The initial result for $\mathcal{E}_{4}$ depends on the Neumann frequencies $w_{1}$ and $w_{2}$. We find it convenient to replace the frequencies by the Jacobi modulus $t$ and the second charge $\mathcal{E}_{2}$. To this end we recall [3, 团 that for the folded string the periodicity condition and the charge $\mathcal{E}_{2}$ written in terms of the w's are (cf. eq.(3.5) and eq.(3.21) )

$$
\mathrm{K}(t)^{2}=\frac{\pi^{2}}{4} w_{21}^{2}, \quad \mathcal{E}_{2}^{2}=w_{1}^{2}+t w_{21}^{2} .
$$

\footnotetext{
${ }^{7}$ The higher derivatives, $x_{i}^{\prime \prime}, x_{i}^{\prime \prime \prime}, \ldots$ are expressed via $x_{i}$ and $x_{i}^{\prime \prime}$ by using the equations of motion.
} 
These formulae allow one to eliminate the $w$ 's. We represent the final result as an expansion in inverse powers of $\mathcal{E}_{2}$.

Coming to the next charge $\mathcal{E}_{5}$ it can be shown that its integrand is a total derivative in the variable $\sigma$ and, therefore, $\mathcal{E}_{5}$ vanishes. Evaluation of $\mathcal{E}_{6}$ is similar to $\mathcal{E}_{4}$, the result is

$$
\begin{aligned}
\mathcal{E}_{6} & =\frac{2^{5}}{\pi^{2} \mathcal{E}_{2}} \mathrm{~K}(t)[\mathrm{E}(t)+(t-1) \mathrm{K}(t)] \\
& -\frac{2^{6}}{3 \pi^{4} \mathcal{E}_{2}^{3}} \mathrm{~K}(t)^{3}[(8 t-4) \mathrm{E}(t)+(t-1)(15 t-4) \mathrm{K}(t)] \\
& +\frac{2^{9}}{\pi^{6} \mathcal{E}_{2}^{5}} t(t-1) \mathrm{K}(t)^{5}[\mathrm{E}(t)+(3 t-2) \mathrm{K}(t)]-\frac{5 \cdot 2^{9}}{\pi^{8} \mathcal{E}_{2}^{7}} t^{2}(t-1)^{2} \mathrm{~K}(t)^{8}
\end{aligned}
$$

The expressions for $\mathcal{E}_{4}$ and $\mathcal{E}_{6}$ already suggest a general pattern: All the odd charges are zero while the even charges admit an expansion in odd powers of the inverse second charge.

The conserved charges $\mathcal{E}_{n}$ comprise an all-loop result. To compare with gauge theory we need to extract the one-loop contribution. Assuming $\mathcal{J}=\mathcal{J}_{1}+\mathcal{J}_{2}$ large one first solves the equation for the Jacobi modulus (3.6) by power series expansion $t=t_{0}+t_{2} / \mathcal{J}^{2}+\ldots$. This implies in particular the following relation between the filling fraction $\alpha$ and the modulus $t_{0}$ :

$$
\alpha=\frac{\mathcal{J}_{2}}{\mathcal{J}}=1-\frac{\mathrm{E}\left(t_{0}\right)}{\mathrm{K}\left(t_{0}\right)} .
$$

Then one can easily verify that the non-trivial string charges admit the following large $\mathcal{J}$ expansion:

$$
\mathcal{E}_{n}=\delta_{2, n} \mathcal{J}+\frac{\epsilon_{n}^{(1)}}{\mathcal{J}}+\frac{\epsilon_{n}^{(2)}}{\mathcal{J}^{3}}+\frac{\epsilon_{n}^{(3)}}{\mathcal{J}^{5}}+\ldots
$$

Note that only the second charge scales with $\mathcal{J}$ to leading order. In the expansion (3.27) all the coefficients $\epsilon_{n}^{(k)}$ are functions of $t_{0}$, while $t_{0}$ is determined by eq.(3.26). In particular we have

$$
\epsilon_{2}^{(1)}=\frac{2}{\pi^{2}} \mathrm{~K}\left(t_{0}\right)\left[\mathrm{E}\left(t_{0}\right)-\left(1-t_{0}\right) \mathrm{K}\left(t_{0}\right)\right]
$$

Working out the expansion (3.27) for $\mathcal{E}_{4}$ we find that the expression for $\epsilon_{4}^{(1)}$ coincides up to a numerical coefficient with $\epsilon_{2}^{(1)}: \epsilon_{4}^{(1)}=-8 \epsilon_{2}^{(1)}$. Thus, at order $1 / \mathcal{J}$ the charges $\mathcal{E}_{2}$ and $\mathcal{E}_{4}$ are not independent. This suggests that the following redefinition of the fourth charge should be made

$$
\mathcal{E}_{4} \rightarrow \mathcal{E}_{4}+8\left(\mathcal{E}_{2}-\mathcal{J}\right) .
$$


Indeed, the expansion of the redefined charge starts from the $1 / \mathcal{J}^{3}$ term and we obtain

$$
\mathcal{Q}_{4} \equiv \epsilon_{4}^{(2)}+8 \epsilon_{2}^{(2)}=\frac{16}{\pi^{4}} \mathrm{~K}\left(t_{0}\right)^{3}\left[\left(2 t_{0}-1\right) \mathrm{E}\left(t_{0}\right)+\left(1-4 t_{0}+3 t_{0}^{2}\right) \mathrm{K}\left(t_{0}\right)\right] .
$$

Analogously we find that at orders $1 / \mathcal{J}$ and $1 / \mathcal{J}^{3}$ the charge $\mathcal{E}_{6}$ is a linear combination of the lower charges $\mathcal{E}_{2}$ and $\mathcal{E}_{4}$. Again this redundant information contained in $\mathcal{E}_{6}$ can be removed by redefining it as

$$
\mathcal{E}_{6} \rightarrow \mathcal{E}_{6}+\frac{128}{3}\left(\mathcal{E}_{2}-\mathcal{J}\right)+\frac{22}{3} \mathcal{E}_{4} .
$$

The expansion of the redefined charge starts from $1 / \mathcal{J}^{5}$ and the coefficient reads

$$
\begin{aligned}
\mathcal{Q}_{6} \equiv & \epsilon_{6}^{(3)}+\frac{128}{3} \epsilon_{2}^{(3)}+\frac{22}{3} \epsilon_{4}^{(3)}= \\
& \frac{2^{9}}{3 \pi^{6}} \mathrm{~K}\left(t_{0}\right)^{5}\left[\left(1-6 t_{0}+6 t_{0}^{2}\right) \mathrm{E}\left(t_{0}\right)+\left(t_{0}-1\right)\left(1-8 t_{0}+10 t_{0}^{2}\right) \mathrm{K}\left(t_{0}\right)\right] .
\end{aligned}
$$

Thus, we see that after redefinition the (improved) charges scale homogeneously with inverse powers of $1 / \mathcal{J}$, i.e. like $\mathcal{Q}_{n} / \mathcal{J}^{n-1}$, as was the case for the conserved charges of the one-loop gauge theory.

Concerning the next two charges we just point out that they require the following redefinition

$$
\begin{aligned}
\mathcal{E}_{8} & \rightarrow \mathcal{E}_{8}+\frac{1024}{5}\left(\mathcal{E}_{2}-\mathcal{J}\right)+\frac{201}{5} \mathcal{E}_{4}+\frac{44}{5} \mathcal{E}_{6} \\
\mathcal{E}_{10} & \rightarrow \mathcal{E}_{10}+\frac{32768}{35}\left(\mathcal{E}_{2}-\mathcal{J}\right)+\frac{6922}{35} \mathcal{E}_{4}+\frac{1898}{35} \mathcal{E}_{6}+\frac{74}{7} \mathcal{E}_{8}
\end{aligned}
$$

and the corresponding values of the improved charges are

$$
\begin{aligned}
\mathcal{Q}_{8}=\frac{2^{11}}{\pi^{8}} \mathrm{~K}\left(t_{0}\right)^{7}\left[\left(2 t_{0}-1\right)\left(1-10 t_{0}+10 t_{0}^{2}\right) \mathrm{E}\left(t_{0}\right)\right. & \\
& \left.+\left(t_{0}-1\right)\left(-1+15 t_{0}-45 t_{0}^{2}+35 t_{0}^{3}\right) \mathrm{K}\left(t_{0}\right)\right], \\
\mathcal{Q}_{10}=\frac{2^{17}}{5 \pi^{10}} \mathrm{~K}\left(t_{0}\right)^{9}[(1- & \left.20 t_{0}+90 t_{0}^{2}-140 t_{0}^{3}+70 t_{0}^{4}\right) \mathrm{E}\left(t_{0}\right) \\
& \left.+\left(t_{0}-1\right)\left(1-24 t_{0}+126 t_{0}^{2}-224 t_{0}^{3}+126 t_{0}^{4}\right) \mathrm{K}\left(t_{0}\right)\right] .
\end{aligned}
$$

Now we will demonstrate that the improved large $\mathcal{J}$ string theory charges $\mathcal{Q}_{2 n}$ and the one-loop gauge theory charges $Q_{2 n}$ are actually the same. The charges $\mathcal{Q}_{2 n}$ depend on the string modulus $t_{0}$, while the $Q_{2 n}$ are governed by $q$. Matching $\mathcal{Q}_{2} \equiv \epsilon_{2}^{(1)}$ with $Q_{2}$ requires that the string modulus is related to the gauge theory modulus by a Gauss-Landen transformation [4, 5], which for the folded string solution is of the form

$$
t_{0}=-\frac{(1-\sqrt{1-q})^{2}}{4 \sqrt{1-q}}
$$


Under this transformation one has

$$
\mathrm{K}\left(t_{0}\right)=(1-q)^{1 / 4} \mathrm{~K}(q), \quad \mathrm{E}\left(t_{0}\right)=\frac{1}{2}(1-q)^{-1 / 4} \mathrm{E}(q)+\frac{1}{2}(1-q)^{1 / 4} \mathrm{~K}(q) .
$$

Remarkably this maps not only the energy, but all higher large $\mathcal{J}$ string charges $\mathcal{Q}_{2 n}$ onto the one-loop gauge charges $Q_{2 n}$. Indeed, transforming the first few (cf eqs.(3.30), (3.32)) $\mathcal{Q}_{2 n}$ with (3.33), (3.34) we obtain

$$
\begin{array}{ll}
\mathcal{Q}_{2}=-\frac{1}{4 \pi^{2}} Q_{2}, & \mathcal{Q}_{4}=\frac{3}{8 \pi^{4}} Q_{4}, \quad \mathcal{Q}_{6}=-\frac{5}{12 \pi^{6}} Q_{6}, \\
\mathcal{Q}_{8}=\frac{7}{16 \pi^{8}} Q_{8}, & \mathcal{Q}_{10}=-\frac{9}{20 \pi^{10}} Q_{10}, \quad \ldots .
\end{array}
$$

Up to unessential numerical prefactors the improved "one-loop" string charges $\mathcal{Q}_{n}$ coincide with the gauge theory charges $Q_{n}, c f$ eqs.(2.25) in section 2.2. An all orders proof will be presented in chapter 4 , and the exact matching formula turns out to be

$$
\mathcal{Q}_{2 k}=(-1)^{k} \frac{2 k-1}{4 k \pi^{2 k}} Q_{2 k} .
$$

We note a certain similarity of the higher terms $\epsilon_{2}^{(n)}$ in the expansion of $\mathcal{E}_{2}$, corresponding to the currently unknown eigenvalues of the dilatation operator at higher loops, and the "one-loop" higher charges $\mathcal{Q}_{n}$. For instance,

$$
\epsilon_{2}^{(2)}=-\frac{2}{\pi^{4}} \mathrm{~K}\left(t_{0}\right)^{3}\left[\left(2 t_{0}-1\right) \mathrm{E}\left(t_{0}\right)+\left(1-t_{0}\right)^{2} \mathrm{~K}\left(t_{0}\right)\right]
$$

is similar to but different from $\mathcal{Q}_{4}$ in eq.(3.30) due to the additional term $\epsilon_{4}^{(2)}$. The difference is of course expected, otherwise the higher loop dilatation operator would commute with a higher one-loop charge, i.e., integrability could be extended to higher loops without any deformation, which is certainly not the case.

With the dressed solution $X(\gamma)$ at hand we may ask for the corresponding values of the commuting charges, as we did for the trial solution $X$. Performing a perturbative (in $\gamma$ ) computation of the spins $\mathcal{J}_{i}$ and the first few charges $\mathcal{E}_{n}$ on $X(\gamma)$, we find that neither the spins nor the charges depend on the deformation parameter $\gamma$; higher corrections $Z^{(k)}$ to the trial solution simply do not contribute. Another important point is that $X(\gamma)$ respects the Virasoro constraints. All this shows, in fact, that the dressed and the trial solutions differ in a very mild fashion. In the next section we will use this observation to solve the Bäcklund equations exactly and to find all string commuting charges.

\subsection{Exact generating function of commuting charges in string theory}

Having gained some experience from perturbative calculus we now want to determine an exact (to all orders in $\gamma$ ) generating function for the commuting charges in string theory. A direct approach to sum the series (3.13) seems hopeless due to increasing 
complexity of the coefficients $X^{(k)}$. Therefore we have to better understand the meaning of the Bäcklund transformations, and reformulate the problem in a suitable way.

A first observation is that the Bäcklund transformation "almost" preserves the Neumann form of the trial solution. An easy way to see this is to write the perturbative solution in terms of the complexified embedding coordinates $Z_{i}=X_{2 i-1}+i X_{2 i}$, $i=1,2,3$, where $X_{1}, \ldots, X_{6}$ are the original real variables of the $\mathrm{O}(6)$ sigma model. Then the first few coefficients of the dressed solution are (3.13)

$$
\begin{aligned}
& Z_{i}=x_{i} e^{i w_{i} \tau}, \\
& Z_{i}^{(1)}=\frac{2}{\sqrt{\mathcal{E}_{2}}}\left(\partial_{\sigma}+i w_{i}\right) x_{i} e^{i w_{i} \tau}, \\
& Z_{i}^{(2)}=\frac{2}{\mathcal{E}_{2}}\left(\partial_{\sigma}+i w_{i}\right)^{2} x_{i} e^{i w_{i} \tau}
\end{aligned}
$$

and so on (the higher terms look more complicated). To get these formulae one uses the fact that $\mathcal{E}_{2}$ is an integral for both the sigma model ( $\tau$-independent) and for the Neumann system ( $\sigma$-independent). Due to the phase exponents, $e^{i w_{i} \tau}$, the deformed solution is still of rotating type, but the $\sigma$-dependent coefficients become complex, in contrast to the real variables $x_{i}$ of the trial solution. Thus, the Bäcklund transformation allows one to construct more general solutions of the $\mathrm{O}(6)$ model which correspond to the "complexified" Neumann ansatz:

$$
Z_{i}=r_{i}(\sigma, \gamma) e^{i \alpha_{i}(\sigma, \gamma)} e^{i w_{i} \tau}
$$

where, in addition to the radial coordinates $r_{i}$, the phase shifts $\alpha_{i}$ come into play.

Another important observation comes from the analysis of the phase shifts $\alpha_{i}$ for the first few perturbative coefficients of the trial solution: The phases $\alpha_{i}$ do not depend on $\sigma$, i.e. they are functions of the spectral parameter only. This fact implies that the variables $r_{i}(\sigma, \lambda)$ solve the evolution equations of the Neumann system:

$$
r_{i}^{\prime \prime}=-w_{i}^{2} r_{i}-r_{i} \sum_{j=1}^{3}\left(r_{j}^{\prime 2}-w_{j}^{2} r_{j}^{2}\right) .
$$

Thus, $r_{i}(\sigma, \gamma)$ is a one-parameter family of solutions obeying the initial condition $r_{i}(\sigma, 0)=x_{i}(\sigma)$. On the other hand we already know all two-spin solutions ${ }^{8}$ of the Neumann system corresponding to the folded and the circular strings $\llbracket$ | They indeed form a one-parameter family, where the parameter is related to rigid shifts of the world-sheet variable $\sigma: \sigma \rightarrow \sigma+$ const. Therefore we can immediately write down the generic folded string solution depending on the spectral parameter:

$$
r_{1}(\gamma)=\operatorname{dn}(\mu+\nu, t), \quad r_{2}(\gamma)=\sqrt{t} \operatorname{sn}(\mu+\nu, t), \quad r_{3}(\gamma)=0
$$

\footnotetext{
${ }^{8}$ The Bäcklund transformation preserves all the global charges of the $\mathrm{O}(6)$ model.
} 
where we introduced the concise notation $\mu=\sigma \sqrt{w_{21}^{2}}$. The variable $\nu$ is a yet unknown function of the spectral parameter $\nu \equiv \nu(\gamma)$; however we know that it cannot depend on $\sigma$. Requiring the solution to be periodic we obtain the usual periodicity condition for the folded string which we repeat here for convenience: $\frac{\pi}{2} \sqrt{w_{21}^{2}}=\mathrm{K}(t)$.

Thus we considerably constrained the ansatz for the Bäcklund solution: The only unknowns left are the two phases $\alpha_{1}, \alpha_{2}$ and the shift function $\nu$, all of which depend solely on the spectral parameter $\gamma$. Now we can use the Bäcklund equations (3.11) to determine $\alpha_{1}, \alpha_{2}, \gamma$, and furthermore check the consistency of our ansatz. The corresponding solution is found in appendix A and we use it to obtain the exact generating function for the commuting string charges. The final result reads

$$
\mathcal{E}(\gamma)=\frac{4 \gamma^{3}}{\pi\left(1+\gamma^{2}\right)} \frac{\sqrt{(1-z)(1-t z)}}{\sqrt{z}} \Pi(t z, t)
$$

where the function $z=\operatorname{sn}^{2} \nu$ satisfies

$$
1-\frac{w_{1}^{2}}{w_{21}^{2}} \frac{z}{1-z}-\left(\frac{1-\gamma^{2}}{1+\gamma^{2}}\right)^{2} \frac{1}{1-t z}=0 .
$$

The last equation implies that $z$ can be expanded in even powers of $\gamma$, starting at $\mathcal{O}\left(\gamma^{2}\right)$. Carefully developing the $\gamma$-expansion of the elliptic integral $\Pi$ and eliminating the $w$ 's with the help of eqs.(3.24)

$$
w_{1}=\frac{1}{\pi} \sqrt{\pi^{2} \mathcal{E}_{2}^{2}-4 t \mathrm{~K}(t)^{2}}, \quad w_{2}=\frac{1}{\pi} \sqrt{\pi^{2} \mathcal{E}_{2}^{2}-4(t-1) \mathrm{K}(t)^{2}},
$$

one can check that the formula (3.42) correctly reproduces our previous findings for the first few non-trivial charges $\mathcal{E}_{2}, \mathcal{E}_{4}, \mathcal{E}_{6}$ and so on. It also shows that all odd charges are zero.

To complete the solution we recall that the energy $\mathcal{E}_{2}$ and the modulus $t$ are functions of the spins determined by the following two equations:

$$
\left(\frac{\mathcal{E}_{2}}{\mathrm{~K}(t)}\right)^{2}-\left(\frac{\mathcal{J}_{1}}{\mathrm{E}(t)}\right)^{2}=\frac{4}{\pi^{2}} t, \quad\left(\frac{\mathcal{J}_{2}}{\mathrm{~K}(t)-\mathrm{E}(t)}\right)^{2}-\left(\frac{\mathcal{J}_{1}}{\mathrm{E}(t)}\right)^{2}=\frac{4}{\pi^{2}}
$$

Thus, all string charges are implicit functions of the spins $\mathcal{J}_{i}$.

To better appreciate the result obtained let us note that the function $\mathcal{E}(\gamma)$ depends explicitly on the energy $\mathcal{E}_{2}$. Given the energy $\mathcal{E}_{2}$ and the modulus $t$, all the higher charges are uniquely determined by (3.42). From the point of view of the Neumann integrability this result is expected: the Neumann system has two integrals of motion which are essentially $\mathcal{E}_{2}$ and $t$. These integrals completely constrain the solution (up to a constant shift in $\sigma$ ), and we certainly do not need to use the higher charges in order to derive the energy. All higher charges of the sigma model 
embedding are then determined a posteriori. It is rather intriguing that this structure appears to differ significantly, on a conceptual level, from the one found on the gauge theory side. There all charges, generated by the transfer matrix operator, are required in order to apply the Bethe ansatz and obtain the solution of the spin chain energy spectrum. The final results, when restricted to one-loop, nevertheless match precisely.

Finally let us also note that eqs.(3.44), (3.45) together with eq.(3.26) allows one to rewrite eq. (3.43) in the form

$$
\left(\frac{1-\gamma^{2}}{1+\gamma^{2}}\right)^{2}=\frac{1-t z}{1-z}\left[1-\left(\frac{\mathrm{K}\left(t_{0}\right)-\mathrm{E}\left(t_{0}\right)}{\mathrm{K}(t)-\mathrm{E}(t)}\right)^{2} \frac{\pi^{2}}{4} \frac{\mathcal{J}^{2}}{\mathrm{~K}\left(t_{0}\right)^{2}} z\right]
$$

This formula is most suitable for generating $\gamma$ - and $\mathcal{J}$-expansions of $z=z(\gamma, \mathcal{J})$ and, as the consequence, of $\mathcal{E}(\gamma)$. Indeed, the second formula in (3.45) and eq.(3.26) can be used to determine

$$
t=t_{0}+\frac{t_{2}}{\mathcal{J}^{2}}+\frac{t_{4}}{\mathcal{J}^{4}}+\ldots
$$

where all the coefficients $t_{2 k}, k>0$ are functions of $t_{0}$. Then all the coefficients in the expansion of $z$ appear to depend on $t_{0}$.

The derivation of the generating function for commuting charges related to the circular string is very similar. The exact solution of the corresponding Bäcklund equations is found in appendix A. Remarkably, the generating function for the circular string turns out to be of the identical form (3.42). The only difference lies in the equation for the shift function $\nu$ :

$$
\frac{t w_{1}^{2}}{w_{12}^{2}} z+\left(\frac{1-\gamma^{2}}{1+\gamma^{2}}\right)^{2} \frac{1-t z}{1-z}-1=0, \quad z=\operatorname{sn}^{2} \nu .
$$

This formula is supplemented by the expressions for the w's via the energy and the modulus

$$
w_{1}=\frac{1}{\pi} \sqrt{\pi^{2} \mathcal{E}_{2}^{2}+4(t-1) \mathrm{K}(t)^{2}}, \quad w_{2}=\frac{1}{\pi} \sqrt{\pi^{2} \mathcal{E}_{2}^{2}-4 \mathrm{~K}(t)^{2}}
$$

and by two transcendental equations yielding the energy and the modulus as functions of the spins

$$
\begin{aligned}
& \left(\frac{\mathcal{E}_{2}}{\mathrm{~K}(t)}\right)^{2}-\left(\frac{t \mathcal{J}_{1}}{\mathrm{~K}(t)-\mathrm{E}(t)}\right)^{2}=\frac{4}{\pi^{2}}(1-t), \\
& \left(\frac{\mathcal{J}_{1}}{\mathrm{~K}(t)-\mathrm{E}(t)}\right)^{2}-\left(\frac{\mathcal{J}_{2}}{(1-t) \mathrm{K}(t)-\mathrm{E}(t)}\right)^{2}=\frac{4}{\pi^{2} t} .
\end{aligned}
$$

These expressions together with the formula relating the modulus $t_{0}$ with the filling fraction $\alpha$ for the circular string:

$$
\alpha=\frac{\mathcal{J}_{2}}{\mathcal{J}}, \quad \alpha=1-\frac{1}{t_{0}}\left(1-\frac{\mathrm{E}\left(t_{0}\right)}{\mathrm{K}\left(t_{0}\right)}\right)
$$


allow one to cast the expression (3.48) in the form

$$
\left(\frac{1-\gamma^{2}}{1+\gamma^{2}}\right)^{2}=\frac{1-z}{1-t z}\left[1-\frac{t^{2}}{t_{0}^{2}}\left(\frac{\mathrm{K}\left(t_{0}\right)-\mathrm{E}\left(t_{0}\right)}{\mathrm{K}(t)-\mathrm{E}(t)}\right)^{2} \frac{\pi^{2}}{4} \frac{\mathcal{J}^{2}}{\mathrm{~K}\left(t_{0}\right)^{2}} z\right] .
$$

The explicit "perturbative" matching of the first few charges generated by $\mathcal{E}(\gamma)$ with their gauge theory counterparts is discussed in appendix A.

\section{Matching the entire infinite tower of commuting charges}

We so far derived the exact generating functions of one-loop gauge charges, eq.(2.24) and eq.(2.39), as well as the exact generating functions of (unimproved) string charges, eqs. 3.42),(3.45), (3.46) and eqs. (3.42),(3.49),(3.51), and we showed that the first few charges indeed coincide if we (a) linearly redefine (improve) the string charges, (b) restrict to one-loop and (c) apply a Gauss-Landen transformation. In this final chapter we will apply this three-step procedure to the entire infinite tower of string charges. In other words, we will derive the infinite number of gauge charges from string theory. To be specific we will treat the case of the folded string first, and work out the circular case in appendix A.2.

The idea is to implement the freedom of linear redefinition on a functional level. In particular, we are clearly allowed to replace $\gamma^{2}$ by a new parameter $\mu^{2}$ which can be an arbitrary formal, even power series in $\gamma^{2}$ such that $\mu^{2}=\gamma^{2}+$ $\mathcal{O}\left(\gamma^{4}\right)$. Ideally we would like to find an improved spectral parameter $\mu$ such that the transformed string resolvent eq.(3.42) only generates terms in the combination $\mu^{2 k} \mathcal{J}^{-2 k+1}+\mathcal{O}\left(\mu^{2 k} \mathcal{J}^{-2 k-1}\right)$. The coefficient multiplying $\mu^{2 k}$ would then correspond to the fully improved string charge. Inspection of eq.(3.46) shows that this can be almost (see below) achieved by defining $\mu$ through

$$
1-4 \mu^{2}=\left(\frac{1-\gamma^{2}}{1+\gamma^{2}}\right)^{2}
$$

Eq.(3.46) becomes

$$
\frac{\mu^{2}}{\pi^{2} \mathcal{J}^{2}}=\frac{t z}{4 \pi^{2} \mathcal{J}^{2}}+\left(\frac{\mathrm{E}\left(t_{0}\right)}{4 \mathrm{~K}\left(t_{0}\right) \mathrm{E}(t)}\right)^{2} \frac{z(1-t z)}{1-z},
$$

and we easily verify that the auxiliary parameter $z$ now indeed expands in the improved fashion. Turning our attention to eq.(3.42), we see that we should drop an overall function in $\mu$ which expands like $1+\mathcal{O}\left(\mu^{2}\right)$, as it would again be a source of unwanted, low powers in $1 / \mathcal{J}$ at $\mathcal{O}\left(\mu^{2 k}\right)$ (this is allowed since it once more corresponds to a linear redefinition). This way we find the following improved generating function

$$
\mathcal{E}(\gamma) \rightarrow \tilde{\mathcal{E}}(\mu)=\frac{4 \mu^{3}}{\pi} \frac{\sqrt{(1-z)(1-t z)}}{\sqrt{z}} \Pi(t z, t)
$$


which generates linearly redefined string charges

$$
\tilde{\mathcal{E}}(\mu)=\sum_{k=1}^{\infty} \tilde{\mathcal{E}}_{2 k} \mu^{2 k}
$$

However, in the line preceding eq.(4.1) we wrote "almost" since, unfortunately, due to the overall power $\mu^{3}$ in eq.(4.3), the redefined charges $\tilde{\mathcal{E}}_{2 k}$ now expand as $\sim$ $\mu^{2 k} \mathcal{J}^{-2 k+3}+\mathcal{O}\left(\mu^{2 k} \mathcal{J}^{-2 k+1}\right)$. This is much better as the old resolvent eq.(3.42), but in principle one further linear redefinition of the charges $\tilde{\mathcal{E}}_{2 k} \rightarrow \overline{\mathcal{E}}_{2 k}$ is needed ${ }^{9}$ :

$$
\overline{\mathcal{E}}_{2 k}=\tilde{\mathcal{E}}_{2 k}+4 \frac{2 k-2}{2 k-3} \overline{\mathcal{E}}_{2 k-2} \text {. }
$$

The $\overline{\mathcal{E}}_{2 k}$ are the properly scaling, fully improved string charges, requiring a further functional transform $\tilde{\mathcal{E}}(\mu) \rightarrow \overline{\mathcal{E}}(\mu)$. However, we see from eq. (4.5) that to leading order in $1 / \mathcal{J}$, the "nearly-improved" charges $\tilde{\mathcal{E}}_{2 k}$ are proportional to the "fullyimproved" charges $\overline{\mathcal{E}}_{2 k-2}$ ! We can therefore introduce a large- $\mathcal{J}$ generating function

$$
\mathcal{Q}(u)=\lim _{\mathcal{J} \rightarrow \infty} \frac{1}{2} \frac{1-\mu^{-2} \tilde{\mathcal{E}}(\mu)}{\mathcal{J}} \quad \text { with } \quad u^{2}=-\frac{\mu^{2}}{\pi^{2} \mathcal{J}^{2}},
$$

where we deliberately use the same notation $u$ as in chapter 2 since it will become clear in a moment that $u$ can indeed be identified with the spectral parameter of the gauge theory resolvent. Restriction to "one-loop" corresponds to keeping $u$ finite and sending $\mathcal{J}$ to infinity. From eq.(4.3) the generating function in (4.6) becomes

$$
\mathcal{Q}(u)=\frac{1}{2}\left[1-4 u \frac{\sqrt{(1-z)\left(t_{0} z-1\right)}}{\sqrt{z}} \Pi\left(t_{0} z, t_{0}\right)\right] .
$$

Furthermore, in this limit eq.(4.2) simplifies drastically and we get

$$
u^{2}+\frac{1}{16 \mathrm{~K}\left(t_{0}\right)^{2}} \frac{z\left(1-t_{0} z\right)}{1-z}=0 .
$$

We pick up the root which in the vicinity of $u \sim 0$ behaves as $z \sim u^{2}$, namely

$$
z=\frac{1-16 \mathrm{~K}\left(t_{0}\right)^{2} u^{2}}{2 t_{0}}\left(1-\sqrt{1+\frac{64 t_{0} \mathrm{~K}\left(t_{0}\right)^{2} u^{2}}{\left(1-16 \mathrm{~K}\left(t_{0}\right)^{2} u^{2}\right)^{2}}}\right) .
$$

The final step consists in applying the Gauss-Landen transformation (3.33) to eq.(4.7). In particular, one can prove that the following modular transformation formula is valid

$$
\Pi\left(t_{0} z, t_{0}\right)=\frac{2 \sqrt{a b}}{4 a b+(a-b)^{2} v}\left[\frac{1}{4}+\frac{a^{2}}{b} \sqrt{\frac{b^{2}-u^{2}}{a^{2}-u^{2}}} \Pi\left(-q \frac{u^{2}}{a^{2}-u^{2}}, q\right)\right] .
$$

\footnotetext{
${ }^{9}$ Our derivation only requires the observation that $\overline{\mathcal{E}}_{2 k}=\tilde{\mathcal{E}}_{2 k}+c_{k-1} \overline{\mathcal{E}}_{2 k-2}$, which is the general form of the linear transformation mapping the charges $\tilde{\mathcal{E}}_{2 k}$ to the fully-improved charges $\overline{\mathcal{E}}_{2 k}$. Using different arguments one can show that the a priori unknown constants $c_{k}$ are given by $c_{k}=\frac{8 k}{2 k-1}$.
} 
Here $t_{0}$ is related to $q$ by eq. 3.33$)$, the variables $a$ and $b$ are given by eqs.(2.16), and $v$ is an image of the variable $z$ in eq. (4.9) under the Gauss-Landen transformation:

$$
v=2 \frac{u^{2}-a b}{(a-b)^{2}}\left(1-\sqrt{1-\frac{(a-b)^{2} u^{2}}{\left(u^{2}-a b\right)^{2}}}\right) .
$$

With these formulae at hand we find that after application of the Gauss-Landen transformation the large $\mathcal{J}$ folded string spectral curve $\mathcal{Q}(u)$, eq.(4.7), becomes

$$
\mathcal{Q}(u)=H(u)+\frac{\alpha}{2} .
$$

where $H(u)$ is precisely the one-loop folded gauge theory resolvent as written in eq.(2.24), q.e.d.

Note added. After this work was completed we learned that the relation between the Bethe resolvent and the properly defined higher gauge theory charges was independently derived by J. Engquist, J.A. Minahan and K. Zarembo in the interesting paper 27].

\section{Acknowledgments}

The work of G.A. was supported in part by the European Commission RTN programme HPRN-CT-2000-00131 and by RFBI grant N02-01-00695. We would like to thank Niklas Beisert, Sergey Frolov, Jan Plefka, Jorge Russo, Arkady Tseytlin and Kostya Zarembo for useful discussions.

\section{A. Exact solution of the Bäcklund equations and generating function}

Using the ansatz (3.39) for the Bäcklund solution we write down the generating function (3.15)

$$
\left.\mathcal{E}(\gamma)=\gamma \int_{0}^{2 \pi} \frac{d \sigma}{4 \pi} r_{i}(\gamma)\left[\left(1-\gamma^{2}\right) \cos \alpha_{i} x_{i}^{\prime}+\left(1+\gamma^{2}\right) w_{i} \sin \alpha_{i} x_{i}\right)\right]
$$

Here $r_{i}(\gamma)$ is the Bäcklund solution (3.41) and $x_{i}$ is the trial solution: $x_{i}=r_{i}(0)$. As was discussed in the main text, to compute $\mathcal{E}(\gamma)$ one has to find the phases $\alpha_{i}(\gamma)$ and the shift function $\nu(\gamma)$.

\section{A.1 Folded case}

We start with the folded string described by eqs.(3.4); the spectral dependent solution $r_{i}(\gamma)$ is given by (3.41). Consider the normalization condition (3.12)

$$
\cos \alpha_{1} r_{1}(\gamma) x_{1}+\cos \alpha_{2} r_{2}(\gamma) x_{2}=\frac{1-\gamma^{2}}{1+\gamma^{2}}
$$


Specifying $\sigma=0$ or $\sigma=\frac{\pi}{2}$ one finds

$$
\cos \alpha_{1}=\frac{1-\gamma^{2}}{1+\gamma^{2}} \frac{1}{\operatorname{dn} \nu}, \quad \cos \alpha_{2}=\frac{1-\gamma^{2}}{1+\gamma^{2}} \frac{\operatorname{cn} \nu}{\operatorname{dn} \nu}
$$

Plugging the found solution back into eq.(A.2) we obtain the following relation

$$
\operatorname{dn}(\mu+\nu) \operatorname{dn} \nu+t \operatorname{cn} \mu \operatorname{sn} \mu \operatorname{sn}(\mu+\nu)=\operatorname{dn} \nu, \quad \mu \equiv \sigma \sqrt{w_{21}^{2}}
$$

which must hold for any value of $\sigma$. This is indeed the case as one can verify by using the addition formulas for elliptic functions.

It is convenient to separate the real and the imaginary parts of the Bäcklund equations. Then one can show that with the choice (A.3) the real part of eqs.(3.11) is identically satisfied while the imaginary part implies the relations

$$
\sin \alpha_{1}=\frac{w_{1}}{\sqrt{w_{21}^{2}}} \frac{\operatorname{sn} \nu}{\operatorname{cn} \nu}, \quad \sin \alpha_{2}=\frac{w_{2}}{\sqrt{w_{21}^{2}}} \operatorname{sn} \nu .
$$

Now the identity $\cos ^{2} \alpha_{1}+\sin ^{2} \alpha_{1}=1$ gives an equation for the unknown function $\nu$ :

$$
\frac{w_{1}^{2}}{w_{21}^{2}} \frac{\operatorname{sn}^{2} \nu}{\operatorname{cn}^{2} \nu}+\left(\frac{1-\gamma^{2}}{1+\gamma^{2}}\right)^{2} \frac{1}{\operatorname{dn}^{2} \nu}=1
$$

which is equivalent to eq.(3.43).

Finally using eqs. A.3 and (A.4) one can prove that the $\sigma$-dependence of the eqs.(3.11) is identically satisfied due to elliptic function identities. This shows consistency of our ansatz for the Bäcklund solution. Thus we have found the exact solution of the Bäcklund equations.

It is worth stressing that the Bäcklund solution we found has the same dual CFT operator as the trial solution. Indeed, the phases in eq.(3.39), which are independent of $\tau$ and $\sigma$, can be gauged away by the rigid $\mathrm{U}(1) \times \mathrm{U}(1) \times \mathrm{U}(1)$ rotation, while the function $\nu(\gamma)$ can be removed by the constant shift of $\sigma$ (the leftover reparametrization invariance). However, the Bäcklund equations themselves are not invariant w.r.t. these symmetries and that is why they lead to a particular solution with non-trivial $\gamma$-dependence.

To obtain the the generating function the following integration formulae are helpful

$$
\begin{aligned}
& \int_{0}^{2 \pi} \frac{d \sigma}{4 \pi} r_{1}(\lambda) r_{1}(0)=\frac{\mathrm{dn} \nu}{2 \mathrm{~K}(t) \mathrm{sn}^{2} \nu}\left[\mathrm{K}(t)-\mathrm{cn}^{2} \nu \Pi\left(t \mathrm{sn}^{2} \nu, t\right)\right], \\
& \int_{0}^{2 \pi} \frac{d \sigma}{4 \pi} r_{2}(\lambda) r_{2}(0)=\frac{\mathrm{cn} \nu \mathrm{dn} \nu}{2 \mathrm{~K}(t) \operatorname{sn}^{2} \nu}\left[\Pi\left(t \mathrm{sn}^{2} \nu, t\right)-\mathrm{K}(t)\right], \\
& \int_{0}^{2 \pi} \frac{d \sigma}{4 \pi} r_{1}(\lambda) r_{1}^{\prime}(0)=\frac{\mathrm{cn} \nu}{\pi \mathrm{sn}^{3} \nu}\left[\mathrm{dn}^{2} \nu\left(\mathrm{K}(t)-\Pi\left(t \operatorname{sn}^{2} \nu, t\right)\right)+\operatorname{sn}^{2} \nu(\mathrm{K}(t)-\mathrm{E}(t))\right], \\
& \int_{0}^{2 \pi} \frac{d \sigma}{4 \pi} r_{2}(\lambda) r_{2}^{\prime}(0)=\frac{1}{\pi \operatorname{sn}^{3} \nu}\left[\operatorname{sn}^{2} \nu \mathrm{E}(t)-\mathrm{dn}^{2} \nu \mathrm{K}(t)+\operatorname{cn}^{2} \nu \operatorname{dn}^{2} \nu \Pi\left(t \operatorname{sn}^{2} \nu, t\right)\right] .
\end{aligned}
$$


They can be easily derived by using the addition formulae for elliptic functions and the integral representation for the complete elliptic integral of the third kind $\Pi(u, t)$. Using these formulae we obtain

$$
\begin{aligned}
\mathcal{E}(\gamma) & =\frac{\gamma}{\pi\left(1+\gamma^{2}\right)}\left[\left(1-\gamma^{2}\right)^{2} \frac{\mathrm{cn} \nu}{\operatorname{sn} \nu \operatorname{dn} \nu}\left[\mathrm{K}(t)-\operatorname{dn}^{2} \nu \Pi\left(t \operatorname{sn}^{2} \nu, t\right)\right]\right. \\
& \left.+\left(1+\gamma^{2}\right)^{2}\left[\frac{\operatorname{dn} \nu \operatorname{cn} \nu}{\operatorname{sn} \nu} \Pi\left(t \operatorname{sn}^{2} \nu, t\right)-\left(\frac{w_{2}^{2} \operatorname{cn} \nu}{w_{21}^{2}}-\frac{w_{1}^{2}}{w_{21}^{2} \operatorname{cn} \nu}\right) \frac{\operatorname{dn} \nu}{\operatorname{sn} \nu} \mathrm{K}(t)\right]\right]
\end{aligned}
$$

The last step consists in expressing the quantities $w_{1}^{2} / w_{21}^{2}$ and $w_{2}^{2} / w_{21}^{2}$ from eq. A.5.

$$
\begin{aligned}
& \frac{w_{1}^{2}}{w_{21}^{2}}=\frac{\mathrm{cn}^{2} \nu}{\operatorname{sn}^{2} \nu}-\left(\frac{1-\gamma^{2}}{1+\gamma^{2}}\right)^{2} \frac{\mathrm{cn}^{2} \nu}{\operatorname{sn}^{2} \nu \operatorname{dn}^{2} \nu}, \\
& \frac{w_{2}^{2}}{w_{21}^{2}}=\frac{1}{\operatorname{sn}^{2} \nu}-\left(\frac{1-\gamma^{2}}{1+\gamma^{2}}\right)^{2} \frac{\mathrm{cn}^{2} \nu}{\operatorname{sn}^{2} \nu \operatorname{dn}^{2} \nu}
\end{aligned}
$$

and substituting them into eq.(A.6). In this way we obtained our final result (3.42).

\section{A.2 Circular case}

Here we will also treat the circular string (3.7), i.e. we will find its curve and charges on the string side and then match it to the gauge theory results in section 2.2. The presentation will be more succinct as the one above.

One starts with the following ansatz for the Bäcklund solution

$$
r_{1}(\gamma)=\operatorname{sn}(\mu+\nu), \quad r_{2}(\gamma)=\operatorname{cn}(\mu+\nu), \quad r_{3}(\gamma)=0 \quad \text { with } \quad \mu=\sigma \sqrt{\frac{w_{12}^{2}}{t}} .
$$

Then the following formulae solve the Bäcklund equations for the circular string:

$$
\begin{aligned}
\cos \alpha_{1} & =\frac{1-\gamma^{2}}{1+\gamma^{2}} \frac{\operatorname{dn} \nu}{\operatorname{cn} \nu}, & \cos \alpha_{2} & =\frac{1-\gamma^{2}}{1+\gamma^{2}} \frac{1}{\operatorname{cn} \nu}, \\
\sin \alpha_{1} & =\frac{\sqrt{t} w_{1}}{\sqrt{w_{12}^{2}}} \operatorname{sn} \nu, & \sin \alpha_{2} & =\frac{\sqrt{t} w_{2}}{\sqrt{w_{12}^{2}}} \frac{\operatorname{sn} \nu}{\operatorname{dn} \nu} .
\end{aligned}
$$

The equation for the shift function $\nu$ is

$$
\frac{t w_{1}^{2}}{w_{12}^{2}} \operatorname{sn}^{2} \nu+\left(\frac{1-\gamma^{2}}{1+\gamma^{2}}\right)^{2} \frac{\operatorname{dn}^{2} \nu}{\operatorname{cn}^{2} \nu}=1
$$

which can be written as

$$
\frac{t w_{1}^{2}}{w_{12}^{2}} z+\left(\frac{1-\gamma^{2}}{1+\gamma^{2}}\right)^{2} \frac{1-t z}{1-z}-1=0, \quad z=\operatorname{sn}^{2} \nu
$$

The frequencies $w_{i}$ are found from the equations [1]

$$
\frac{\pi^{2}}{4} \frac{w_{12}^{2}}{t}=\mathrm{K}(t)^{2}, \quad \mathcal{E}_{2}^{2}=w_{2}^{2}+\frac{w_{12}^{2}}{t}, \quad w_{1}>w_{2}
$$


and they are

$$
w_{1}=\frac{1}{\pi} \sqrt{\pi^{2} \mathcal{E}_{2}^{2}+4(t-1) \mathrm{K}(t)^{2}}, \quad w_{2}=\frac{1}{\pi} \sqrt{\pi^{2} \mathcal{E}_{2}^{2}-4 \mathrm{~K}(t)^{2}} .
$$

The exact generating function is

$$
\begin{aligned}
\mathcal{E}(\gamma)= & \frac{\gamma}{\pi\left(1+\gamma^{2}\right)}\left[\left(1-\gamma^{2}\right)^{2} \frac{\operatorname{dn} \nu}{\operatorname{sn} \nu \operatorname{cn} \nu}\left[\mathrm{K}(t)-\operatorname{cn}^{2} \nu \Pi\left(t \operatorname{sn}^{2} \nu\right)\right]\right. \\
& +\left(1+\gamma^{2}\right)^{2}\left[\frac{\operatorname{cn} \nu \operatorname{dn} \nu}{\operatorname{sn} \nu} \Pi\left(t \operatorname{sn}^{2} \nu, t\right)-\left(\frac{w_{1}^{2} \operatorname{dn} \nu}{w_{12}^{2}}-\frac{w_{2}^{2}}{w_{12}^{2} \operatorname{dn} \nu}\right) \frac{\operatorname{cn} \nu}{\operatorname{sn} \nu} \mathrm{K}(t)\right]
\end{aligned}
$$

Again, excluding the $w$ 's with the help of eq. A.12) for the shift function $\nu$ we can simplify the expression (A.15) to get

$$
\mathcal{E}(\gamma)=\frac{4 \gamma^{3}}{\pi\left(1+\gamma^{2}\right)} \frac{\operatorname{cn} \nu \operatorname{dn} \nu}{\operatorname{sn} \nu} \Pi\left(t \operatorname{sn}^{2} \nu, t\right)
$$

Quite remarkably, the generation function for the circular string turns out to be exactly of the same form as as the one of the folded string. The difference lies of course in the equation for the shift function.

As in the folded string case all odd charges vanish for the circular string configuration. From eq.(A.16) it is easy to obtain the explicit expressions for the charges, e.g. the first two non-trivial charges $\mathcal{E}_{4}$ and $\mathcal{E}_{6}$ are

$$
\begin{aligned}
\mathcal{E}_{4} & =-\frac{16}{\pi^{2} \mathcal{E}_{2}} \mathrm{E}(t) \mathrm{K}(t)-\frac{32}{\pi^{4} \mathcal{E}_{2}^{3}}(t-1) \mathrm{K}(t)^{4}, \\
\mathcal{E}_{6} & =\frac{2^{5}}{\pi^{2} \mathcal{E}_{2}} \mathrm{E}(t) \mathrm{K}(t)+\frac{2^{6}}{3 \pi^{4} \mathcal{E}_{2}^{3}} \mathrm{~K}(t)^{3}[4(t-2) \mathrm{E}(t)+7(t-1) \mathrm{K}(t)] \\
& -\frac{2^{9}}{\pi^{6} \mathcal{E}_{2}^{5}}(t-1) \mathrm{K}(t)^{5}[\mathrm{E}(t)-(t-2) \mathrm{K}(t)]-\frac{5 \cdot 2^{9}}{\pi^{8} \mathcal{E}_{2}^{7}}(t-1)^{2} \mathrm{~K}(t)^{8} .
\end{aligned}
$$

For the same reason as for the folded string the higher charges $\mathcal{E}_{n}$ evaluated on the circular type solution need to be improved in order to scale homogeneously. It turns out that the corresponding redefined charges are given by the same formulae (3.29) and (3.31). This is not surprising since a universal relation between the string and the gauge theory models implies that the charge redefinition must be universal as well, i.e., it should be independent of particular solutions of the evolution equations.

String theory provides the following values for the improved charges at one loop:

$$
\begin{aligned}
& \mathcal{Q}_{2}=\frac{2}{\pi^{2}} \mathrm{~K}\left(t_{0}\right) \mathrm{E}\left(t_{0}\right), \\
& \mathcal{Q}_{4}=-\frac{16}{\pi^{4}} \mathrm{~K}\left(t_{0}\right)^{3}\left[\left(t_{0}-2\right) \mathrm{E}\left(t_{0}\right)+\left(t_{0}-1\right) \mathrm{K}\left(t_{0}\right)\right], \\
& \mathcal{Q}_{6}=\frac{2^{9}}{3 \pi^{6}} \mathrm{~K}\left(t_{0}\right)^{5}\left[\left(6-6 t_{0}+t_{0}^{2}\right) \mathrm{E}\left(t_{0}\right)+2\left(2-3 t_{0}+t_{0}^{2}\right) \mathrm{K}\left(t_{0}\right)\right],
\end{aligned}
$$




$$
\begin{gathered}
\mathcal{Q}_{8}=\frac{2^{11}}{\pi^{8}} \mathrm{~K}\left(t_{0}\right)^{7}\left[\left(2-t_{0}\right)\left(10-10 t_{0}+t_{0}^{2}\right) \mathrm{E}\left(t_{0}\right)-3\left(t_{0}-1\right)\left(5-5 t_{0}+t_{0}^{2}\right) \mathrm{K}\left(t_{0}\right)\right], \\
\mathcal{Q}_{10}=\frac{2^{17}}{5 \pi^{10}} \mathrm{~K}\left(t_{0}\right)^{9}\left[\left(70-140 t_{0}+90 t_{0}^{2}-20 t_{0}^{3}+t_{0}^{4}\right) \mathrm{E}\left(t_{0}\right)\right. \\
\left.+4\left(14-35 t_{0}+30 t_{0}^{2}-10 t_{0}^{3}+t_{0}^{4}\right) \mathrm{K}\left(t_{0}\right)\right]
\end{gathered}
$$

and so on. The string charge $\mathcal{Q}_{2}$ and the gauge charge $Q_{2}$ in eq.(2.32)) are related by the following Gauss-Landen transformation

$$
t_{0}=-\frac{4 \sqrt{r}}{(1-\sqrt{r})^{2}} .
$$

This results in

$$
\mathrm{K}\left(t_{0}\right)=(1-\sqrt{r}) \mathrm{K}(r), \quad \mathrm{E}\left(t_{0}\right)=2(1-\sqrt{r})^{-1} \mathrm{E}(r)-(1+\sqrt{r}) \mathrm{K}(r)
$$

The same transformation applies to all higher charges. It is easy to check that under this transformation the string charges again (A.19) nicely turn into the gauge charges (2.32), 2.40), up to a multiplicative constant:

$$
\begin{aligned}
& \mathcal{Q}_{2}=-\frac{1}{4 \pi^{2}} Q_{2}, \quad \mathcal{Q}_{4}=-\frac{3}{8 \pi^{4}} Q_{4}, \quad \mathcal{Q}_{6}=-\frac{5}{12 \pi^{6}} Q_{6}, \\
& \mathcal{Q}_{8}=-\frac{7}{16 \pi^{8}} Q_{8}, \quad \mathcal{Q}_{10}=-\frac{9}{20 \pi^{10}} Q_{10}, \quad \ldots .
\end{aligned}
$$

The general formula is

$$
\mathcal{Q}_{2 k}=-\frac{2 k-1}{4 k \pi^{2 k}} Q_{2 k}
$$

Up to the factor $(-1)^{k+1}$ (see the comment after eq. (2.37)) this formula coincides with eq.(3.36).

Finally, the proof of the exact matching of the gauge and the "one-loop" string spectral curves for the circular string configuration proceeds in analogy to the treatment in chapter 4 . Let us briefly sketch the main steps. Introducing the improved spectral parameter

$$
x^{2}=\frac{\mu^{2}}{\pi^{2} \mathcal{J}^{2}},
$$

where $\mu$ is defined through eq.(4.1), we find from eq.(3.51) the following relation in the large $\mathcal{J}$ limit:

$$
x^{2}-\frac{1}{16 \mathrm{~K}\left(t_{0}\right)^{2}} \frac{z(1-z)}{1-t_{0} z}=0 .
$$


The large $\mathcal{J}$ circular string spectral curve is then found from eq.(3.42) to be

$$
\mathcal{Q}(x)=\frac{1}{2}\left[1-4 x \frac{\sqrt{(1-z)\left(1-t_{0} z\right)}}{\sqrt{z}} \Pi\left(t_{0} z, t_{0}\right)\right] .
$$

The slight difference with eq.(4.7) is due to our sign convention for the charges (once again see the comment after eq. (2.37)). The Gauss-Landen transformation (A.20) is applied by using the transformation rule $(x \leq c<d)$

$$
\Pi\left(t_{0} z, t_{0}\right)=\frac{d-c}{c d+x^{2}+\sqrt{\left(c^{2}-x^{2}\right)\left(d^{2}-x^{2}\right)}}\left[\frac{1}{8}+\frac{1}{d} \sqrt{\left(c^{2}-x^{2}\right)\left(d^{2}-x^{2}\right)} \Pi\left(\frac{x^{2}}{d^{2}}, r\right)\right],
$$

where $c$ and $d$ are given by eqs.(2.30). The result we find is

$$
\mathcal{Q}(x)=H(x)-\frac{\alpha}{2},
$$

where $H(x)$ is precisely the circular gauge theory spectral curve in eq.(2.39), q.e.d.

\section{A.3 The Gauss-Landen transformation}

It is of interest to recall the geometric meaning of the Gauss-Landen transformation and to understand what it means in our present context. The Jacobi modulus $t$ completely defines an elliptic curve $y^{2}=\left(1-x^{2}\right)\left(1-t x^{2}\right)$, in particular, its complex structure $\tau$ which is the ratio of the primitive periods $\omega_{1}$ and $\omega_{2}: \tau=\frac{\omega_{2}}{\omega_{1}}$. The curve can be uniformized by the function sn, i.e. $y=\operatorname{sn}^{\prime} z, x=\operatorname{sn} z$, which has the periods $\omega_{1}=4 \mathrm{~K}(t)$ and $\omega_{2}=2 i \mathrm{~K}(1-t)$. Thus, the complex structure is

$$
\tau=\frac{\omega_{2}}{\omega_{1}}=\frac{i}{2} \frac{\mathrm{K}(1-t)}{\mathrm{K}(t)}
$$

For the string curve defining the circular string solution in the one-loop approximation the complex structure $\tau_{s}$ is therefore

$$
\tau_{s}=\frac{i}{2} \frac{\mathrm{K}\left(1-t_{0}\right)}{\mathrm{K}\left(t_{0}\right)} .
$$

Taking into account eqs.(A.21) and the transformation rule

$$
\mathrm{K}\left(1-t_{0}\right)=\frac{1}{2}(1-\sqrt{r}) \mathrm{K}(1-r)
$$

we perform the Gauss-Landen transformation (A.20) on $\tau_{s}$ :

$$
\tau_{s}=\frac{i}{2} \frac{\mathrm{K}\left(1-t_{0}\right)}{\mathrm{K}\left(t_{0}\right)}=\frac{i}{4} \frac{\mathrm{K}(1-r)}{\mathrm{K}(r)}=\frac{1}{2} \tau_{g}
$$

where $\tau_{g}$ appears to be the complex structure of the elliptic curve governing the position of the Bethe roots on the gauge theory side. We therefore realize that the 
gauge curve ${ }^{10}$ defining the Bethe root distribution and the string curve which solves the string evolution equations are related in a simple way: the former is a doublecover of the latter. A similar interpretation might be given for the folded string solution.

The potential importance of our observation about the interrelation between the string and the gauge curves is that the covering index (two in our case) is an integer and, therefore, it might be stable to loop deformations, i.e. it could survive even if the higher loops are concerned. This would mean that the Gauss-Landen transformation is universal and it could be extended to higher loops as well.

\section{B. Conventions for elliptic integrals and elliptic functions}

For the benefit of the serious reader we are collecting our conventions for various elliptic integrals and functions.

The complete elliptic integrals of the first $(\mathrm{K})$ and second $(\mathrm{E})$ kind are

$$
\mathrm{K}(q) \equiv \int_{0}^{\pi / 2} \frac{d \varphi}{\sqrt{1-q \sin ^{2} \varphi}} \quad \mathrm{E}(q) \equiv \int_{0}^{\pi / 2} d \varphi \sqrt{1-q \sin ^{2} \varphi}
$$

and the complete elliptic integral of the third kind is

$$
\Pi\left(m^{2}, q\right) \equiv \int_{0}^{\pi / 2} \frac{d \varphi}{\left(1-m^{2} \sin ^{2} \varphi\right) \sqrt{1-q \sin ^{2} \varphi}}
$$

To derive the string spectral curve the following integral representation for $\Pi\left(m^{2}, q\right)$ was used

$$
\int_{0}^{2 \pi} \frac{d \sigma}{1-m^{2} \operatorname{sn}^{2}\left(\frac{2}{\pi} \mathrm{K}(q) \sigma, q\right)}=\frac{2 \pi}{\mathrm{K}(q)} \Pi\left(m^{2}, q\right)
$$

The Jacobi elliptic function $\operatorname{sn} \nu \equiv \operatorname{sn}(\nu, q)$ inverts the elliptic integral of the first kind:

$$
\nu=\int_{0}^{\operatorname{sn} \nu} \frac{d x}{\sqrt{\left(1-x^{2}\right)\left(1-q x^{2}\right)}}
$$

The elliptic functions $\operatorname{cn} \nu \equiv \operatorname{cn}(\nu, q)$ and $\operatorname{dn} \nu \equiv \operatorname{dn}(\nu, q)$ are related to $\operatorname{sn} \nu$ through the identities:

$$
\operatorname{sn}^{2} \nu+\mathrm{cn}^{2} \nu=1, \quad \operatorname{dn}^{2} \nu+q \operatorname{sn}^{2} \nu=1
$$

\footnotetext{
${ }^{10}$ The curves we discussed here should not be confused with the spectral curves, the latter depend on the additional spectral parameter and generate the infinite family of commuting charges.
} 


\section{References}

[1] S. Frolov and A. A. Tseytlin, "Multi-spin string solutions in $A d S_{5} \times S^{5}$," Nucl. Phys. B 668 (2003) 77, hep-th/0304255.

[2] N. Beisert, J. A. Minahan, M. Staudacher and K. Zarembo, "Stringing spins and spinning strings," JHEP 0309 (2003) 010, hep-th/0306139.

[3] S. Frolov and A. A. Tseytlin, "Rotating string solutions: AdS/CFT duality in non-supersymmetric sectors," Phys. Lett. B 570 (2003) 96, hep-th/0306143.

[4] G. Arutyunov, S. Frolov, J. Russo and A. A. Tseytlin, "Spinning strings in $A d S_{5} \times S^{5}$ and integrable systems," hep-th/0307191.

[5] N. Beisert, S. Frolov, M. Staudacher and A. A. Tseytlin, "Precision spectroscopy of $A d S / C F T, "$ hep-th/0308117.

[6] D. Berenstein, J. M. Maldacena and H. Nastase, "Strings in flat space and pp waves from $N=4$ super Yang Mills," JHEP 0204 (2002) 013, hep-th/0202021.

[7] S. Frolov and A. A. Tseytlin, "Semiclassical quantization of rotating superstring in $A d S_{5} \times S^{5}$," JHEP 0206 (2002) 007, hep-th/0204226.

[8] S. Frolov and A. A. Tseytlin, "Quantizing three-spin string solution in $A d S_{5} \times S^{5}$," JHEP 0307 (2003) 016, hep-th/0306130.

[9] H. J. de Vega, A. L. Larsen and N. Sanchez, "Semiclassical quantization of circular strings in de Sitter and anti-de Sitter space-times," Phys. Rev. D 51 (1995) 6917, hep-th/9410219.

[10] S. S. Gubser, I. R. Klebanov and A. M. Polyakov, "A semi-classical limit of the gauge/string correspondence," Nucl. Phys. B 636 (2002) 99, hep-th/0204051.

[11] J. A. Minahan and K. Zarembo, "The Bethe-ansatz for $N=4$ super Yang-Mills," JHEP 0303, (2003) 013, hep-th/0212208.

[12] N. Beisert, "The complete one-loop dilatation operator of $N=4$ super Yang-Mills theory," hep-th/0307015.

[13] N. Beisert and M. Staudacher, "The $N=4 S Y M$ integrable super spin chain," hep-th/0307042.

[14] N. Beisert, C. Kristjansen and M. Staudacher, "The dilatation operator of $N=4$ super Yang-Mills theory," Nucl. Phys. B 664 (2003) 131, hep-th/0303060; see also N. Beisert, "Higher loops, integrability and the near BMN limit," hep-th/0308074.

[15] D. Mateos, T. Mateos and P. K. Townsend, "Supersymmetry of tensionless rotating strings in $A d S_{5} \times S^{5}$, and nearly-BPS operators," hep-th/0309114.

[16] A. Gorsky, "Spin chains and gauge / string duality," hep-th/0308182. 
[17] G. Mandal, N. V. Suryanarayana and S. R. Wadia, "Aspects of semiclassical strings in $A d S_{5}$," Phys. Lett. B 543 (2002) 81, hep-th/0206103.

[18] I. Bena, J. Polchinski and R. Roiban, "Hidden symmetries of the $A d S_{5} \times S^{5}$ superstring," hep-th/0305116.

[19] L. Dolan, C. R. Nappi and E. Witten, "A relation between approaches to integrability in superconformal Yang-Mills theory," hep-th/0308089.

[20] L. F. Alday, "Non-local charges on $A d S_{5} \times S^{5}$ and pp-waves," hep-th/0310146.

[21] K. Pohlmeyer, "Integrable Hamiltonian systems and interactions through quadratic constraints," Commun. Math. Phys. 46, 207 (1976).

[22] I. V. Cherednik, "Local conservation laws of principal chiral fields $(D=1)$," Theor. Math. Phys. 38 (1979) 120 [Teor. Mat. Fiz. 38 (1979) 179]; "Conservation laws and elements of scattering theory for principal chiral fields $(D=1)$," Theor. Math. Phys. 41 (1979) 997 [Teor. Mat. Fiz. 41 (1979) 236].

[23] A. T. Ogielski, M. K. Prasad, A. Sinha and L. L. Wang, "Bäcklund transformations and local conservation laws for principal chiral fields," Phys. Lett. B 91 (1980) 387.

[24] H. J. De Vega and N. Sanchez, "Exact integrability of strings in D-Dimensional De Sitter space-time," Phys. Rev. D 47 (1993) 3394.

[25] I. K. Kostov and M. Staudacher, "Multicritical Phases Of The O(N) Model On A Random Lattice," Nucl. Phys. B 384 (1992) 459, hep-th/9203030.

[26] O. Babelon and D. Bernard, "Affine solitons: a relation between tau functions, dressing and Bäcklund transformations,” Int. J. Mod. Phys. A 8 (1993) 507, hep-th/9206002.

[27] J. Engquist, J. A. Minahan and K. Zarembo, "Yang-Mills duals for semiclassical strings on $A d S_{5} \times S^{5}$," hep-th/0310188. 